\title{
Some results in the effective descriptive set theory ${ }^{* *}$
}

\author{
Dedicated to Professor Motokiti Kondô \\ on his sixtieth birthday anniversary
}

By

Hisao TANAKA*

\section{Contents}

§0. Introduction.

$\S 1$. Preliminaries.

§. Examples.

§3. Non-denumerable $\sum_{1}^{1}$-sets.

$\$ 4$. The measure of an arithmetical set.

$\S 5$. The measure of a $\sum_{1}^{1}$-sets and related problems.

$\S 6$. $\Pi_{1}^{1}$-sets containing perfect subsets.

$\$ 7$. Concerning a basis result for the arithmetical sets.

§8. On the Baire's theorem with respect to a monotone wellordered family of closed sets.

\section{$\S 0 . \quad$ Introduction}

Since about 1955, one has investigated the descriptive set theory from the standpoint of the effective hierarchy. Such a theory is called "effective descriptive set theory" by Addison. Many results appeared in this branch (e.g., [1-4],[15],[27-28],[32],[33-35],[36],[37]). The present paper belongs to the same branch. In this theory, several classical theorems can be very easily proved, sometimes by using results

Received November 10, 1966.

Communicated by T. Takasu.

* College of Engineering, Hôsei University.

** This paper contains the contents of the author's [35] with detailed proofs and also further results. 
in the recursive function theory. The main reasons are based on full use of logical smybols and on the consideration of the character of an individual real number itself (such as being (hyper) arithmetically real). Such examples will be stated in $\$ 2$ below. To some sets and ordinals whose existence had been proved classically, we shall determine their positions which they occupy in the effective hierarchy. For instance, it is well-known that every non-denumerable analytic set contains a perfect subset.1) For this, we can obtain Theorem 2: Every nondenumerable $\sum_{1}^{1}$ set in $N^{N}$ contains a perfect subset $P$ which is $\Pi_{1}^{1}$ recursively closed, and hence it is in the class $\Delta_{2}^{1}$. (For notations and terminology, see the following sections.) Since there is a nondenumerable $\sum_{1}^{1}$-set which contains no non-void $\Pi_{1}^{1}$-subsets (Theorem 3 ), such a $\mathrm{P}$ cannot be (in general) a $\Pi_{1}^{1}$-set. The above results will be proved in $\S 3$.

Concerning the Lebesgue measure, one knows that every analytic set is Lebesgue measurable. Kreisel [18] shows that the measure of an arithmetical set in the segment $I=[0,1]$ is an arithmetical real number. (Cf. Grzegorczyk [6].) It is proved that this fact also remains true when we consider arithmetical sets in $N^{N}$ instead of those in $I$ (Corollary 3). What sort of real number is the measure of a $\sum_{1}^{1}$ set in $N^{N}$ ? To this question we answer by Theorem 8 as follows: The measure of a $\sum_{1}^{1}$-set is a $\sum_{1}^{1}$ real number in a suitable sense. And hence it is seen that the measure of every $\Delta_{1}^{1}$-set is a $\Delta_{1}^{1}$ real number (in the usual sense). The former gives an improvement of Sampei [28; Theorem 5]. - Some of our results are closely related to and overlapped with those of Sampei [28]. But both were obtained almost independently. - As applications of Theorem 8 we shall give respective versions of Selivanowski's theorem concerning $C A$ sets [29] and of Kondô-Tugué's theorem [14]. These are the main contents of $\$ \$ 4-5$.

In $\$ 6$ we shall deal a $\Pi_{1}^{1}$-set containing a perfect subset. There we shall obtain an effective variant of Lusin's result [24], asserting

1) Here and after "perfect" means "non-empty perfect". 
that one can choose a perfect subset in a $C A$-set having a non-denumerable constituent. In $\S 7$. we obtain a result concerning a basis thcorem for the arithmetical sets in $N^{N}$, that is theorem 16: If $E$ is an arithmetical set and mes $(E)>0$, then $E$ contains an arithmetical point. When $E$ is $\Pi_{1}^{0}$ (in a hyperarithmetical function), this theorem is contrasted to Kleene [8; XXVI]. (See Remark 4 below.) Finally, in $\S 8$ we shall obtain an effective version of Baire's theorem with respect to a monotone well-ordered family of closed (open) sets. Namely let $\mathscr{I}$ be a $\sum_{1}^{1}$-family of open sets in $I$. (For its definition, see $\$ 8$ below.) If $\mathscr{I}$ is well-ordered by the inclusion relation $\subseteq$, then its order-type represents a constructive ordinal (Theorem 18). This theorem will be proved by using the following proposition (Corollary 5): Let $E$ be a $\sum_{1}^{1}$-set in $N^{N}$. If $E$ is well-ordered with respect to the usual linear ordering of real numbers, then its ordertype represents a constructive ordinal.

I wish express my hearty thanks to Professor Motokiti Kondô for his valuable suggestions and kind encouragement in the preparation of this paper.

\section{$\S 1$. Preliminaries}

1.1. Notations and terminology. Let $N$ and $N^{N}$ be the set of all natural numbers and the set of all 1-place number-theoretic functions, respectively. The lower case Roman letters mostly are used as variables ranging over $N$ or constants of $N$, if nothing is said to the contrary. The lower case Greek letters (except for $\xi$ and $\eta$, which are reserved to represent ordinal numbers) are used as constants of or variables over $N^{N}$. We identify $N^{N}$ with the Baire's zero-space and often do so a sequence number $\bar{\alpha}(k)(\neq 1)$ with a Baire's interval $\delta_{n_{1} \cdots n_{k}}$ where $n_{i}=\alpha(i-1)+1$. Notation and terminology for the recursive function theory are ones in Kleene [7-10] if we do not indicate otherwise. Following Addison [3], we denote by $\sum_{n}^{r}$ or $\Pi_{n}^{r}$ the class of sets (or predicates) expressible in the respective forms in Kleene's (arithmetical for $r=0$ and analytic for $r=1$ ) hierarchy, according as 
the outermost quantifier is the existential or the universal one. After Shoenfield we put $\Delta_{n}^{r}=\sum_{n}^{r} \cap \Pi_{n}^{r}$.

Let $\{\delta(1), \delta(2), \cdots, \delta(n), \cdots\}$ and $\left\{\delta^{k}(1), \delta^{k}(2), \cdots, \delta^{k}(n), \cdots\right\}$ be standard effective enumerations of all sequence numbers $(\neq 1)$ and of all sequence numbers of length $k>0$ without repetition, respectively. Of course we may assume $\lambda k n \delta^{k}(n)$ is recursive. When we regard $\{\delta(n)\}_{n>0}$ and $\left\{\delta^{k}(n)\right\}_{n>0}$ as sequences of Baire's intervals, we add $\delta(0)=$ $\delta^{k}(0)=\phi$ (=the empty set) to them. Let $I$ denote the closed interval $[0,1]$ and $\left\{I_{n}\right\}_{n>0}$ denote an effective enumeration of all open intervals $\left(r_{n}, s_{n}\right)$ with rational endpoints contained in $I$ without repetition. Further let $I_{0}=\phi$ and $r_{0}=s_{\mathrm{c}}=0$. When we consider sets in the space $I$, "open" and "closed" are understood relative to $I$.

1.2. We shall often use the following the uniformization theorem - which was first proved by Kondô in the classical case - and/or the effective choice principle of its special case: ${ }^{2)}$

Uniformization Theorem (Addison [2], Kondô [11], Sampei [26], Suzuki [32]). Every $\Pi_{1}^{1}$-set in $N^{N} \times N^{N}$ can be made uniform by a set of the same class.

Effective Choice Principle. From every non-empty $\Pi_{1}^{1}$-set in $N^{N}$ one can choose a point $\alpha$ such that the one element set $\{\alpha\}$ is also a $\Pi_{1}^{1}$-set; and hence $\alpha$ itself is a $\Delta_{2}^{1}$ number-theoretic function (i.e., its representing predicate can be expressed in a $\Delta_{2}^{1}$-form).

\section{§2. Examples}

In this paragraph we shall give several examples which are easily proved by the recursuve function theoretic method rather than the classical descriptive set theoretic one.

2.1. Let $D$ be a set in the product space $X \times Y$, where $X=Y=$ $N^{N}$, and let $D^{\langle\alpha\rangle}$ denote the set $\{\beta \mid\langle\alpha, \beta\rangle \in D\}$. The set, which consists of all points $\alpha$ such that $D^{\prime \alpha\rangle}$ are not well-ordered by the usual linear

2) The method of using this principle was suggested by Professor Y. Sampei to the author. Also cf. [28]. 
ordering $\leq^{3)}$ with respect to the positive direction of the $Y$-axis, is called the set determined by the sieve $D$.

(A). The set $E$ determined by a $\sum_{1}^{1}$-sieve $D$ is also a $\sum_{1}^{1}$ set. (Lusin [23; p. 180])

Proof. We can describe the predicate " $\alpha \in E$ " as follows:

$$
\alpha \in E \equiv(\exists \beta)(x)\left[\beta_{x} \in D^{\prime \alpha} \wedge \beta_{x+1}<\beta_{\lambda}\right],
$$

where $\beta_{x}(t)=\beta(J(x, t))$ and $J$ is a (fixed) usual pairing function. Hence $E$ is $\sum_{11}^{1}$, since $\beta_{x} \in D^{\langle\alpha\rangle}$ is $\sum_{1}^{1}$. (Q.E.D.)

The relativized version of (A) implies the classical one.

2.2. Let $D$ be a $\sum_{1}^{1}$-set in $X \times Y$.

(B) If $E=\left\{\alpha \mid D^{\prime \alpha\rangle}\right.$ is not scattered $\}$, then $E$ is a $\sum_{1}^{1}$-set. (Lusin [23; p. 182])

Proof. It holds the following equivalences:

$\alpha \in E \equiv D^{\langle\alpha\rangle}$ contains a subset dense in itself,

$\equiv D^{\prime \alpha\rangle}$ contains a countable subset dense in itself,

$\equiv \quad(\exists \beta)\left[(n)\left(\beta_{n} \in D^{\langle\alpha\rangle}\right) \wedge\left(\right.\right.$ the set $\left\{\beta_{n} \mid n \geq 0\right\}$ is dense in itself)].

But

$\left\{\beta_{n} \mid n \geq 0\right\}$ is dense in itself $\equiv(n)(\exists \gamma)\left[\lim _{k \rightarrow \infty} \beta_{\gamma(k)}=\beta_{n}\right]$. Hence $E$ is $\sum_{11}^{1}$. (Q.E.D.)

2.3. It is well-known that there is a $G_{\delta^{-}}$set which can be made uniform by no analytic set. Corresponding to this we have:

(C) There is a $\Pi_{1}^{0}$-set in $X \times Y$ which can be made uniform by no $\sum_{1}^{1}$ set.

Proof.4) Gandy [5] proved that there is a recursive $K_{0}(u, v)$ such that

$$
\begin{aligned}
& (\alpha)(\exists \beta)(x) K_{0}(\bar{\alpha}(x), \bar{\beta}(x)) ; \\
& (\alpha)(\overline{\exists \beta}) \mathscr{H}_{\mathcal{E}[\alpha]}(x) K_{0}(\bar{\alpha}(x), \bar{\beta}(x)),
\end{aligned}
$$

3) $\alpha<\beta \equiv \mathrm{B}(x)[\bar{\alpha}(x)=\bar{\beta}(x) \wedge\{(2 \mid x \wedge \alpha(x)>\beta(x)) \vee \overline{(2 \mid x} \wedge \alpha(x)<\beta(x))\}]$.

4) This proof is due to Dr. Y. Suzuki. He obtained it as an effective counterpart of a classical result of P. Novikov. 
where the index $\mathscr{H}[\alpha]$ indicates that the quantifier is restricted to the set $\mathscr{H}[a]$, which is the set of all 1 -place number-theoretic functions hyperarithmetical in $\alpha$. Let $E$ be a set $N^{N} \times N^{N}$ defined by

$$
\langle\alpha, \beta\rangle \in E \equiv(x) K_{0}(\bar{\alpha}(x), \bar{\beta}(x)) \text {. }
$$

$E$ is a disired set. For, suppose $E$ were uniformized by a $\sum_{1}^{1}$-set $B$ :

$$
\begin{aligned}
& (\exists \beta)[\langle\alpha, \beta\rangle \in E] \rightarrow(\exists ! \beta)[\langle\alpha, \beta\rangle \in B], \\
& B \subseteq E .
\end{aligned}
$$

Take a recursive function $\alpha_{0}$. Then $B^{\left\langle\alpha_{0}\right\rangle}$ is also $\sum_{1}^{1}$, and by (1) and (4) it consists of a single point, say $\beta_{0}$. Hence, clearly $\beta_{0}$ is hyperarithmetical. But since $B \subseteq E$, by (2) $\beta_{0}$ cannot be hyperarithmetical, and hence we have a contradiction.

2.4. Lusin proved a classical analytic set in $X \times Y$ can be made uniform by a $A_{\rho \sigma \delta}$-set (cf. [22; p. 66]). Watanabe [38] showed that it can be uniformized by the complement of a $A_{\rho \sigma}$-set. But these proofs are rather complicated and/or geometrical. We can obtain a more simpler proof of the above result.

(D) Every $\sum_{1}^{1}$-set in $X \times Y$ can be uniformized by the complement of a $\left(\sum_{1}^{1}\right)_{\rho \sigma}$-set. ${ }^{5)}$

To prove this proposition, we use a basis result of Kleene [6]. He showed that

$$
(\exists \alpha)(x) K(\bar{\alpha}(x)) \rightarrow(x) K(\bar{\gamma}(x)),
$$

where $\left.\gamma(a)=(\mu k)(\exists \alpha)(x) K\left(\bar{\gamma}(a) * 2^{k+1} * \bar{\alpha}(x)\right){ }^{6}\right)$ We remark that if $K$ is recursive then the function $r$ is in the class $\left(\sum_{1}^{1}\right)_{\rho \sigma} \cap C\left(\sum_{1}^{1}\right)_{\rho \sigma}$.

5) A set $E$ is said to be a $\left(\sum_{1}^{1}\right)_{\rho}$-set, if it is the difference of two $\sum_{1}^{1}$-sets. A set $E$ is said to be a $\left(\sum_{1}^{1}\right)_{\rho \sigma}$-set, if it is expressible in the following form:

$$
\alpha \in E \equiv(\exists n)[P(\alpha, n) \wedge Q(\alpha, n)] \text {, }
$$

where $P$ and $Q$ are in the classes $\sum_{1}^{1}$ and $\Pi_{1}^{1}$, respectively. We shall denote by $C\left(\sum_{1}^{1}\right)_{\rho \sigma}$ the class of all sets whose complements belong to the class $\left(\sum_{1}^{1}\right)_{\rho \sigma}$.

6) This corresponds to considering the following system of sets in the classical analytic set theory:

$$
E^{n_{0} n_{1} \cdots n_{k}}=\bigcup_{\nu \in N^{N}} \prod_{i=0}^{\infty} E_{n_{\nu} r_{1} \cdot . . n_{R^{\nu}}(0) \nu(1) \cdots \nu(i)}
$$

where $E=\bigcup_{\nu \in N^{N}} \bigcap_{i=0}^{\infty} E_{\nu(0) \nu(1) \ldots \nu(i)}$ is a given analytic set. 
Now let

$$
Q(\gamma, \beta) \equiv(\exists \alpha)(x) R(\gamma, \bar{\beta}(x), \bar{\alpha}(x)),
$$

where $R(r, u, v)$ is recursive. Following Kleene [8] and Tugué-Tanaka [37] we define

$$
\begin{aligned}
& \operatorname{Seq}_{2}(w) \equiv \operatorname{Seq}(w) \wedge w=\prod_{t<l h(w)} p_{t} \exp \left(2^{\left((w)_{t}-1\right)_{0}} \cdot 3^{((w) t: 1)_{1}}+1\right), \\
& w_{j}=\prod_{t<l h(w)} p_{t}^{\left((w)_{t}: 1\right)_{j}+1} \quad \text { for } j=0,1
\end{aligned}
$$

and

$$
K(r, w) \equiv \operatorname{Seq}_{2}(w) \wedge R\left(r, w_{c}, w_{1}\right)
$$

Then we have

$$
(\exists \beta)(\exists \alpha)(x) R(\gamma, \bar{\beta}(x), \bar{\alpha}(x)) \equiv(\exists \alpha)(x) K(\gamma, \bar{\alpha}(x)) .
$$

Now we shall define the partial functional $\Psi$ as follows:

$$
\Psi\langle\gamma\rangle(a) \simeq(\mu k)(\exists \alpha)(x) K\left(r, \overline{\Psi\langle\gamma\rangle}(a) * 2^{k+1} * \overline{\boldsymbol{\alpha}}(x)\right) .
$$

Then $\Psi$ satisfies the following condition:

$$
(\exists \beta) Q(\gamma, \beta) \rightarrow(x)[\Psi\langle\gamma\rangle(x) \text { is defined } \wedge K(\gamma, \overline{\Psi\langle\gamma\rangle}(x))] .
$$

Let $U(\gamma, \beta)$ be defined by the following equivalence:

$$
U(\gamma, \beta) \equiv(\exists \alpha) Q(\gamma, \alpha) \wedge(x)(y)\left[\Psi\langle\gamma\rangle(x)=y \rightarrow(y)_{0}=\beta(x)\right] .
$$

By (7) - (8) and the above remark, $\Psi\langle\gamma\rangle(x)=y$ is expressible of a $C\left(\sum_{1}^{1}\right)_{\rho \sigma}$-form; and hence so is $U(\gamma, \beta)$. Further the $U$ satisfies the following conditions:

$$
\begin{aligned}
& (\exists \beta) Q(\gamma, \beta) \rightarrow(\exists ! \beta) U(\gamma, \beta), \text { and } \\
& U(\gamma, \beta) \rightarrow Q(\gamma, \beta),
\end{aligned}
$$

by $(6)-(9)$. Consequently the set $\hat{\gamma} \hat{\beta} U(\gamma, \beta)$ is a $C\left(\sum_{1}^{1}\right)_{\rho \sigma}$-uniformizator of the $\sum_{1}^{1}$-set $\hat{\gamma} \widehat{\beta} Q(\gamma, \beta)$. (Q.E.D.)

2.5. Tugué-Tanaka [37] obtained a simple proof of a result which implies Lusin's “Theorème sur la projection d'ensemble d'unicité”. 


\section{§3. Non-denumerable $\sum_{1}^{1}$-sets}

3.1. Regularization of a given Souslin's system. Let $E$ be a $\sum_{1^{-}}^{1}$ subset of $N^{N}$ defined as follows:

$$
\alpha \in E \equiv(\exists \beta)(x) R_{0}(\bar{\alpha}(x), \bar{\beta}(x)),
$$

where $R_{0}(u, v)$ is a recursive number-theoretic predicate. Following Mostowski we define

$$
\operatorname{rst}(w, x)= \begin{cases}\prod_{i<x} p_{i}^{(w)_{i}} & \text { if } x \leq \ln (w) \\ 0 & \text { otherwise }\end{cases}
$$

Then "rst" is recursive and

$$
i \leq x \rightarrow \operatorname{rst}(\bar{\alpha}(x), i)=\bar{\alpha}(i)
$$

holds. Now let

$$
\begin{aligned}
R_{1}(u, w) \equiv u & =w=1 \vee\{\operatorname{Seq}(u) \wedge \operatorname{Seq}(w) \wedge \operatorname{lh}(u)=\operatorname{lh}(w)>0 \\
& \left.\wedge(i)_{i \leq l h(u)} R_{0}(\operatorname{rst}(u, i), \operatorname{rst}(w, i))\right\} .
\end{aligned}
$$

$R_{1}$ is also recursive and satisfies (1) and (2) below:

$$
\begin{aligned}
& \alpha \in E \equiv(\exists \beta)(x) R_{1}(\bar{\alpha}(x), \bar{\beta}(x)), \text { and } \\
& R_{1}(\bar{\alpha}(x+1), \bar{\beta}(x+1)) \rightarrow R_{1}(\bar{\alpha}(x), \bar{\beta}(x)),
\end{aligned}
$$

Further let $u_{n}^{(w)}$ be defined as follows:

$$
\left\{\begin{array}{l}
u_{n}^{(w)}=\delta^{l h(w)}(n+1) \quad \text { if } \overline{(\exists u)} R_{1}(u, w) \\
u_{0}^{(w)}=(\mu u) R_{1}(u, w) \quad \text { if }(\exists u) R_{1}(u, w) \\
u_{n+1}^{(w)}= \begin{cases}(\mu u)\left[u>u_{n}^{(w)} \wedge R_{1}(u, w)\right] \quad \text { if } \quad(\exists u)\left[u>u_{n}^{(w)} \wedge R_{1}(u, w)\right] \\
u_{n}^{(w)} & \text { if }(\exists u) R_{1}(u, w) \wedge \overline{(\exists u)}\left[u>u_{n}^{(w)} \wedge R_{1}(u, w)\right]\end{cases}
\end{array}\right.
$$

By (3) we have

$$
(\exists u) R_{1}(u, w) \rightarrow(n) R_{1}\left(u_{n}^{(w)}, w\right) .
$$

We shall define the functions $f, g, h$ and a predicate $R_{2}$ in the following way:

$$
g(x, w)=K_{1}\left((w)_{x} \dot{-1}\right), h(x, w)=K_{2}\left((w)_{x} \dot{-1}\right),{ }^{7)}
$$

7) $K_{1}$ and $K_{2}$ are the recursive functions that satisfy the condition $J\left(K_{1}(x), K_{2}(x)\right)$ $=x$. 


$$
\begin{aligned}
& \bar{g}(k ; w)=\prod_{i<k} p_{i}^{g(i, w)+1}, \\
& f(u, w, 0)= \begin{cases}s g|u-1| & \text { if } w=1, \\
1 & \text { otherwise, }\end{cases} \\
& f(u, w, 1)= \begin{cases}s g \mid u-u_{h(0, w)}^{(\bar{g}(1: w))} \text { if } \operatorname{Seq}(w) \wedge l h(w)=1, \\
1 & \text { otherwise. }\end{cases}
\end{aligned}
$$

For $k \geq 1$,

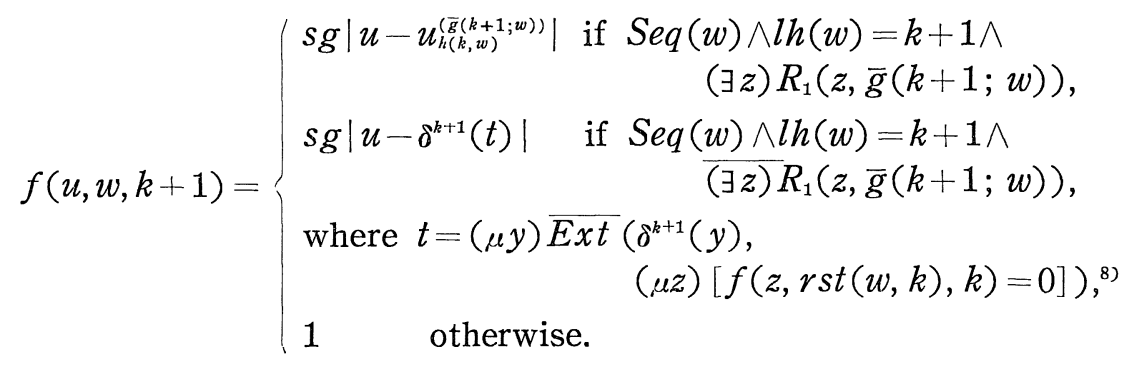

$$
\begin{aligned}
& R_{2}(u, w) \equiv f(u, w, \ln (w))=0 .
\end{aligned}
$$

Then obviously the function $f$ is arithmetical and hence so is $R_{2}$. And $R_{2}$ has the following properties:

(i) $R_{2}(1,1)$ is true,

(ii) if $\overline{\operatorname{Seq}}(u) \vee \overline{\operatorname{Seq}(w)} \vee \operatorname{lh}(u) \neq \operatorname{lh}(w)$, then $R_{2}(u, w)$ is false, and

(iii) $\quad(w)\left[\operatorname{Se} q(w) \rightarrow(\exists ! u) R_{2}(u, w)\right]$.

Lemma 1. $\alpha \in E \equiv(\exists \beta)(k) R_{2}(\bar{\alpha}(k), \bar{\beta}(k))$.

Proof. Suppose $\alpha \in E$. By (1) there is a function $\beta$ such that $(k) R_{1}(\bar{\alpha}(k), \bar{\beta}(k))$. By the definition of $u_{n}^{(w)}$ we can find a sequence $\left\{m_{k}\right\}$ such that

$$
u_{m_{k-1}}^{(\bar{\beta}(k))}=\overline{\boldsymbol{\alpha}}(k) \text { for } k \geq 1 .
$$

Take $\gamma(k)=J\left(\beta(k), m_{k}\right)$. Then $\bar{g}(k ; \bar{\gamma}(k))=\bar{\beta}(k)$ and $h(k-1, \bar{\gamma}(k))$ $=m_{k-1}$. By the definition of $R_{2}$ and by (5) we have

$$
R_{2}(u, \bar{\gamma}(k)) \equiv u=\bar{\alpha}(k) \quad \text { for } \quad k \geq 1 \text {. }
$$

Since $R_{2}(\bar{\alpha}(0), \bar{\gamma}(0))$ is true, it holds

8) $\quad \operatorname{Ext}(u, w) \equiv \operatorname{Seq}(u) \wedge \operatorname{Seq}(w) \wedge w=r s t(u, \operatorname{lh}(w))$. 
(k) $R_{2}(\bar{\alpha}(k), \bar{\gamma}(k))$.

Hence we have $(\exists \gamma)(k) R_{2}(\bar{\alpha}(k), \bar{r}(k))$. Conversely, suppose there is a function $r$ such that (6) holds. Take $\beta(k)=K_{1}(\gamma(k))$. First we shall prove:

$$
\text { (k) ( } \exists) R_{1}(u, \bar{\beta}(k)) \text {. }
$$

Assume there is an $x>0$ such that $\overline{(\exists u) R_{1}}(u, \bar{\beta}(x))$. By (2) we would have $(\exists u) R_{1}(u, \bar{\beta}(x+1))$. Hence, since $R_{2}(\bar{\alpha}(x+1), \quad \bar{\gamma}(x+1))$ $\equiv \bar{a}(x+1)=\delta^{x+1}(t)$, where

$$
t=(\mu y) \overline{E x t}\left(\delta^{x+1}(y),(\mu z) R_{2}(z, \bar{\gamma}(x))\right),
$$

we would have

$$
\bar{\alpha}(x+1)=\delta^{x+1}(t) .
$$

By the above (iii) and (6)

$$
(\mu z) R_{2}(z, \bar{\gamma}(x))=\bar{\alpha}(x)
$$

and hence by (8) it would be

$$
\overline{\operatorname{Ext}}\left(\delta^{x+1}(t), \bar{\alpha}(x)\right) .
$$

This contradicts (9). Hence we must have (7). By (7) and the definition of $R_{2}$ the following equivalence holds:

$$
R_{2}(u, \bar{\gamma}(k)) \equiv u=u_{h(k-1, \bar{\gamma}(k))}^{(\bar{\beta}(k)} \quad \text { for } k \geq 1 .
$$

By our assumption (6) it holds

$$
k>0 \rightarrow \bar{\alpha}(k)=u_{h(k-1, \bar{\gamma}(k))}^{(\bar{\beta}(k)} .
$$

On the other hand, by (4) and (7)

$$
(k)_{k=0}(n) R_{1}\left(u_{n}^{(\bar{\beta}(k))}, \bar{\beta}(k)\right),
$$

especially

$$
(k)_{k>0} R_{1}\left(u_{h(k-1,1)}^{(\bar{\beta}(k))}, \bar{\beta}(k)\right) .
$$

Hence by (10) we have $(k)_{k}{ }_{0} R_{1}(\bar{\alpha}(k), \bar{\beta}(k))$. Consequently it is proved $(\exists \beta)(k) R_{1}(\bar{\alpha}(k), \bar{\beta}(k))$, since $R_{1}(\bar{\alpha}(0), \bar{\beta}(0))$ is true. Thus $\alpha \in E$. (Q.E.D.) 
Now we shall define $R$ as follows:

$$
\begin{array}{r}
R(u, w) \equiv \operatorname{Seq}(u) \wedge \operatorname{Seq}(w) \wedge l h(u)=\operatorname{lh}(w) \wedge \\
(i)_{i \leq l h(u} R_{2}(r s t(u, i), \operatorname{rst}(w, i)) .
\end{array}
$$

Lemma 2. The predicate $R(u, w)$ is arithmetical and satisfies the following conditions:

$$
\begin{aligned}
& \alpha \in E \equiv(\exists \beta)(k) R(\bar{\alpha}(k), \bar{\beta}(k)) . \\
& R(1,1) \text { is true. }
\end{aligned}
$$

or consists of a single Barie's interval of order $k$.

$$
R(\bar{\alpha}(k+1), \bar{\beta}(k+1)) \rightarrow R(\bar{\alpha}(k), \bar{\beta}(k)) .
$$

Proof is obvious by Lemma 1 and (11).

\subsection{The evaluation of the predicate $|E|>*_{0}$}

Theorem 1. Let $E$ be a $\sum_{1}^{1}$-set. Then $|E|>*_{0}$ is expressible in a $\sum_{1}^{1}$-form.

Proof.9) Let $E$ be defined by

$$
\alpha \in E \equiv(\exists \beta)(x) R_{0}(\bar{\alpha}(x), \bar{\beta}(x)),
$$

where $R_{0}$ is recursive. By Lemma 2 we can find an arithmetical predicate $R$ such that (12)-(15) hold. Then $|E|>*_{0}$ can be expressed in the following form:

$$
|E|>\aleph_{0} \equiv(\exists \beta) Q(\beta),
$$

where

$$
\begin{aligned}
& Q(\beta) \equiv(k)\{\operatorname{Seq}(\beta(k)) \wedge(\exists u) R(u, \beta(k))\} \wedge \\
& \text { (k) }(i)\{k \neq i \wedge \operatorname{lh}(\rho(k))=\operatorname{lh}(\rho(i)) \rightarrow
\end{aligned}
$$

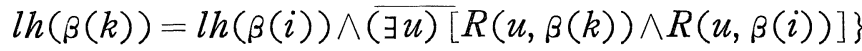

$$
\begin{aligned}
& \wedge(k)(i)\{\operatorname{Ext}(\rho(k), \rho(i)) \rightarrow \operatorname{Ext}(\beta(k), \beta(i)))\} \text {, }
\end{aligned}
$$

and

$$
\rho(0), \rho(1), \rho(2), \cdots
$$

9) We shall use the method of proof in Kondô [13]. 
is an effective enumeration (without repetition) of all sequence number consisting of 0 and 1 only. We prove (16): Suppose there is a function $\beta$ such that $Q(\beta)$. Let $M(\alpha, w)$ be defined as follows:

$M(\alpha, w) \equiv\left\{\begin{array}{c}R(\bar{\alpha}(l), \beta(\mu k)[w=\rho(k)])) \quad \text { if }(\exists k)[w=\rho(k)], \\ \quad \text { where } l=\operatorname{lh}(\beta((\mu k)[w=\rho(k)])), \\ 0 \neq 0 \text { otherwise. }\end{array}\right.$

Then we have

$$
\operatorname{Ext}(w, v) \wedge M(\alpha, w) \rightarrow M(\alpha, v),
$$

and

$$
\text { ( } \nu)\left[\nu \in 2^{N} \rightarrow(\exists ! \alpha)(k) M(\alpha, \bar{\nu}(k)) \wedge(\alpha)\{(k) M(\alpha, \bar{\nu}(k)) \rightarrow \alpha \in E\}\right] .
$$

In fact, let $w=\rho(k), v=\rho\left(k^{\prime}\right), l=\operatorname{lh}(\beta(k))$ and $l^{\prime}=\operatorname{lh}\left(\beta\left(k^{\prime}\right)\right)$. By the premise of (18), $\operatorname{Ext}\left(\rho(k), \rho\left(k^{\prime}\right)\right)$ holds. Hence by (17), $\operatorname{Ext}(\beta(k)$, $\left.\beta\left(k^{\prime}\right)\right)$. Since $M(\alpha, w)$, we have $R(\bar{\alpha}(l), \beta(k))$. Thus $R\left(\bar{\alpha}\left(l^{\prime}\right), \beta\left(k^{\prime}\right)\right)$ by (15). This proves (18). Next, we show (19): For each $\nu \in 2^{N}$ and $k$ there is a number $q_{k}$ and a function $r$ such that $\bar{\nu}(k)=\rho\left(q_{k}\right)$ and (k) $\left[\beta\left(q_{k}\right)=\bar{\gamma}\left(m_{k}\right)\right]$ by (17), where $m_{k}=\operatorname{lh}\left(\beta\left(q_{k}\right)\right)$. Obviously

$$
k<i \rightarrow k \leq m_{k}<m_{i} .
$$

Since $(k)(\exists u) R\left(u, \beta\left(q_{k}\right)\right)$, we have $(k)(\exists u) R\left(u, \bar{\gamma}\left(m_{k}\right)\right)$. Hence by (20), (14) and (15)

$$
(\exists ! \alpha)(k) R\left(\bar{\alpha}\left(m_{k}\right), \bar{\gamma}\left(m_{k}\right)\right) \text {. }
$$

Again by (15) and (20)

$$
\text { (k) } R\left(\bar{\alpha}\left(m_{k}\right), \bar{\gamma}\left(m_{k}\right)\right) \equiv(k) R(\bar{\alpha}(k), \bar{\gamma}(k)) .
$$

So, the unique $\alpha$ such that $(k) R\left(\bar{\alpha}\left(m_{k}\right), \bar{\gamma}\left(m_{k}\right)\right)$ holds belongs to the set $E$ (by (12)). Since $M(\alpha, \bar{\nu}(k)) \equiv R\left(\bar{\alpha}\left(m_{k}\right), \bar{\gamma}\left(m_{k}\right)\right)$, (19) holds.

Now we shall show

$$
\nu, \tau \in 2^{N} \wedge \bar{\nu}(k) \neq \bar{\tau}(k) \rightarrow \overline{(\exists \alpha)}[M(\alpha, \bar{\nu}(k)) \wedge M(\alpha, \bar{\tau}(k))] .
$$

Let $q_{k}$ and $r_{k}$ be the numbers such that $\bar{\nu}(k)=\rho\left(q_{k}\right)$ and $\bar{\tau}(k)=\rho\left(r_{k}\right)$, respectively. As $q_{k} \neq r_{k}$ and $\ln \left(\rho\left(q_{k}\right)\right)=\operatorname{lh}\left(\rho\left(r_{k}\right)\right)=k$, we have

$$
\overline{(\exists u)}\left[R\left(u, \beta\left(q_{k}\right)\right) \wedge R\left(u, \beta\left(r_{k}\right)\right)\right] \text {, }
$$


Hence by the definition of $M$

$$
\overline{(\exists \alpha)}[M(\alpha, \bar{\nu}(k)) \wedge M(\alpha, \bar{\tau}(k))] .
$$

Thus, (21) is true. Let

$$
M=\hat{\boldsymbol{\alpha}}(\exists \nu)\left[\nu \in 2^{N} \wedge(k) M(\alpha, \bar{\nu}(k))\right] .
$$

Then by (18), (21) and (19) $M=\hat{\alpha}(k)(\exists \nu)\left[\nu \in 2^{N} \wedge M(\alpha, \bar{\nu}(k))\right]$ and $\phi \neq M \subseteq E$. As the quantifier $(\exists \nu)_{\nu \epsilon^{N}}$ on the right-hand member of (22) can be replaced by a bounded existential number-quantifier with suitable recursive conditions, $M$ is a closed set in the space $N^{N}$. (Call to mind the fact that each set $\hat{\alpha} R(\bar{\alpha}(\operatorname{lh}(\beta(k))), \beta(k))$ is open-closed in $N^{N}$.) Further the set $M$ is dense in itself. For, let $\alpha$ be an arbitrary element of $M$. Then there is a $\nu \in 2^{N}$ such that $(k) M(\alpha, \bar{\nu}(k))$. By (19) $\left(\overline{\exists \alpha^{\prime}}\right)_{\alpha^{\prime} \neq \alpha}(k) M\left(\alpha^{\prime}, \bar{\nu}(k)\right)$. Let $\nu^{\prime}(k)=1 \dot{-} \nu(k)$ and

$$
\gamma_{k}(i)= \begin{cases}\nu(i) & \text { if } i<k, \\ \nu^{\prime}(i) & \text { if } i=k, \\ 0 & \text { otherwise. }\end{cases}
$$

Since $\gamma_{k} \in 2^{N}$, by (19) for each $\mathrm{k}$ there is a unique function $\alpha_{k}$ such that (i) $M\left(\alpha_{k}, \bar{\gamma}_{k}(i)\right)$. Of course $M\left(\alpha_{k}, \bar{\gamma}_{k}(k+1)\right)$, namely $M\left(\alpha_{k}, \bar{\nu}(k) *\left[\nu^{\prime}(k)\right]\right) .^{10)}$ By (18) we have $M\left(\alpha_{k}, \bar{\nu}(k)\right)$. On the other hand, by (21)

$$
\overline{\left(\exists \alpha^{\prime}\right)}\left[M\left(\alpha^{\prime}, \bar{\nu}(k) *[\nu(k)]\right) \wedge M\left(\alpha^{\prime}, \bar{\nu}(k) *\left[\nu^{\prime}(k)\right]\right)\right] .
$$

Hence $\boldsymbol{\alpha}_{k} \neq \boldsymbol{\alpha}$ for all $k$. Further, if $k \neq k^{\prime}$ then $\boldsymbol{\alpha}_{k} \neq \boldsymbol{\alpha}_{k^{\prime}}$. Consequently, $\alpha$ is a limiting point of $M$. Hence $M$ is dense in itself. Thus, $M$ is a perfect subset of $E$. This implies $|E|>*_{0}$.

Conversely, suppose $|E|>*_{0}$. Then for any $\nu \in 2^{N}$ there are different sequence numbers $\delta(\bar{\nu}(k))(k=0,1,2, \cdots)$ such that for each $k$

(i) if $\nu, \tau \in 2^{N}$ and $\bar{\nu}(k) \neq \bar{\tau}(k)$ then $\ln (\delta(\bar{\nu}(k)))=\ln (\delta(\bar{\tau}(k)))$

$$
\wedge(\exists u)[R(u, \delta(\bar{\nu}(k))) \wedge R(u, \delta(\bar{\tau}(k)))],
$$

(ii) $|E \cap \hat{\alpha} R(\operatorname{lh}(\delta(\bar{\nu}(k))), \delta(\bar{\nu}(k)))|>\star_{0}$,

(iii) $\operatorname{Ext}(\delta(\bar{\nu}(k+1)), \delta(\bar{\nu}(k)))$,

10) $\left[a_{0}, a_{1}, \ldots a_{k-1}\right]=\prod_{i<k} p_{i}^{a_{i+1}}$ 
and hence by (15) it holds:

(iv) $R(\bar{\alpha}(\operatorname{lh}(\delta(\bar{\nu}(k+1)))), \delta(\bar{\nu}(k+1)))$

$\rightarrow R(\bar{\alpha}(\ln (\delta(\bar{\nu}(k)))), \delta(\bar{\nu}(k)))$.

Take $\beta(k)=\delta(\rho(k))$. This function $\beta$ satisfies the condition $Q(\beta)$. Thus we have proved (16).

Since $R$ is arithmetical, $|E|>*_{0}$ is expressible of the form $\sum_{1}^{1}$. This completes the proof of theorem 1 .

3.3. A set $P$ in $N^{N}$ is said to be $\Pi_{1}^{1}$-recursively closed if its complement is expressible in the following form:

$$
C P=\bigcup\{\delta(n) \mid n \in Q\},
$$

for some $Q$ recursive in a $\Pi_{1}^{1}$-number-theoretic predicate (cf. Kreisel [17]).

Theorem 2. For every non-denumerable $\sum_{1}^{1}$-set $E$ in $N^{N}$ there exists a perfect subset $P$ of $E$ which is $\Pi_{1}^{1}$-recursively closed. $A$ fortiori, $P$ belongs to the class $\Delta_{2}^{1}$.

Proof. By Lemma 2, we can define an arithmetical predicate $\mathrm{R}$ satisfying the condition (12)-(15). Since $\lambda \alpha w[\alpha \in E \wedge R(\bar{\alpha}(\operatorname{lh}(w))$, $w)]$ is $\sum_{1}^{1}$, by Theorem 1 so is $\left.\lambda w[\mid E \cap \hat{\alpha} R(\bar{\alpha}(\ln (w)), w)]>*_{0}\right]$. Hence there is a recursive function $\varphi$ such that

$$
|E \cap \hat{\alpha} R(\bar{\alpha}(\operatorname{lh}(w)), w)|>*_{0} \equiv \varphi(w) \notin O,
$$

where $O$ is the complete $\Pi_{1}^{1}$-set (of natural numbers) defined in Kleene [7]. Now suppose $|E|>*_{0}$. We shall define the functions $f, g, l$ and sets $M_{n}$ as follows:

$$
\begin{gathered}
\text { Step 0. } f(0)=(\mu w)\left[w=\left\langle(w)_{0},(w)_{1}\right\rangle^{11)} \wedge(i)_{i<2} \operatorname{Seq}\left((w)_{i}\right)\right. \\
\left.\wedge(w)_{0} \neq(w)_{1} \wedge \operatorname{lh}\left((w)_{0}\right)=\operatorname{lh}\left((w)_{1}\right)>2 \wedge(i)_{i<2}\left\{\varphi\left((w)_{i}\right) \notin O\right\}\right], \\
g(0)=(f(0))_{0}, g(1)=(f(0))_{1} \text { and } l(0)=\operatorname{lh}(g(0)) \quad(=\ln (g(1))>2) .
\end{gathered}
$$

By our assumption $|E|>*_{0}, f(0)$ is defined and hence for $i<2$

$$
|E \cap \hat{\alpha} R(\bar{\alpha}(l(0)), g(i))|>*_{0} .
$$

11) $\left\langle a_{0}, a_{1}, \ldots, a_{k-1}\right\rangle=\prod_{i<k} p_{l}^{a_{i}}$ 
Let $\left.M_{0}=\left\{u \mid \operatorname{Seq}(u) \wedge l h(u)=l(0) \wedge(i)_{i<2} R \overline{(u}, g(i)\right)\right\}$.

Step $n+1$. Suppose $f(i), l(i), M_{i}(i \leq n)$ and $g(i)\left(i \leq 2^{n+2}-3\right)$ are defined, and $\left|E \cap \hat{\alpha} R\left(\bar{\alpha}(l(n)), g\left(2^{n+1}-2+i\right)\right)\right|>*_{0}$ for $i \leq 2^{n+1}-1$. Then define

$$
f(n+1)=(\mu w)\left[w=\left\langle(w)_{0}, \quad(w)_{1}, \cdots, \quad(w)_{2^{n+2}-1}\right\rangle \wedge(i)_{i<2^{n+2}}(j)_{j<2^{n+2}}\right.
$$
$\left\{\operatorname{Seq}\left((w)_{i}\right) \wedge \operatorname{lh}\left((w)_{i}\right)=\operatorname{lh}\left((w)_{0}\right)>\operatorname{lh}\left(g\left(2^{n+1}-2\right)\right) \wedge\left(i \neq j \rightarrow(w)_{i} \neq(w)_{j}\right)\right.$ $\left.\left.\wedge \varphi\left((w)_{i}\right) \notin O\right\} \wedge(i)_{i \leq 2^{n+1}-1}(j)_{2 i \leq j \leq 2 i+1} \operatorname{Ext}\left((w)_{j}, g\left(2^{n+1}-2+i\right)\right)\right]$. Obviously $f(n+1)$ is well defined by the hypothesis of induction. Let $g(i)=(f(n+1))_{i-\left(2^{n+2}-2\right)}$ for $2^{n+2}-2 \leq i \leq 2^{n+3}-3$, and $l(n+1)$ $=\ln \left(g\left(2^{n+2}-2\right)\right)\left(=\cdots=\ln \left(g\left(2^{n+3}-3\right)\right)\right)$. Then

$$
|E \cap \hat{\alpha} R(\bar{\alpha}(l(n+1)), g(i))|>*_{0} \text {, if } 2^{n+2}-2 \leq i \leq 2^{n+3}-3 .
$$

Let $M_{n+1}=\{u \mid \operatorname{Seq}(u) \wedge l h(u)=l(n+1)$

$$
\left.\wedge(i)_{2^{n+2}-2 \leq i \leq 2^{n+3}-3} \bar{R}(u, g(i))\right\} \text {. }
$$

Thus we obtain $f, g, l$ and $\left\{M_{n}\right\}$.

We shall regard each $M_{n}$ as a union of Baire's intervals and hence as a subset of $N^{N}$. Now let $M$ and $P$ be defined as follows:

$$
M=\bigcup_{n=0}^{\infty} M_{n} \text { and } P=C M .
$$

Then by the definitions, we see that $P$ is $\Pi_{1}^{1}$-recursively closed. Further let the function $\psi$ be defined recursively as follows:

$$
\begin{aligned}
& \psi(0)=[0], \psi(1)=[1] \text { and for } i \geq 2 \\
& \psi(i)= \begin{cases}\psi([i / 2]-1) *[0] & \text { if } 2 \mid i, \\
\psi([i / 2]-1) *[1] & \text { otherwise, }\end{cases}
\end{aligned}
$$

where $[i / j]$ is the quotient function. The $\psi$ enumerates the $\{0,1\}-$ sequence numbers in the following way:

$$
[0]^{0}
$$$$
[0,0]^{2} \quad[0,1]^{3}
$$$$
\text { . } . \cdots
$$

For $\nu \in 2^{N}$ and $k$ we put

$$
\left.g^{*}(\bar{\nu}(k))=g(\mu i)[i=\psi(\bar{\nu}(k))]\right),
$$


and

$$
E_{\bar{\nu}(n)}=\hat{\alpha} R\left(\bar{\alpha}\left(\operatorname{lh}\left(g^{*}(\bar{\nu}(n))\right)\right), g^{*}(\bar{\nu}(n))\right)
$$

for $\nu \in 2^{N}$. Then

$$
P=\sum_{\nu \equiv 2^{N}} \prod_{n=1}^{\infty} E_{\bar{\nu}(n)}=\prod_{n=1}^{\infty} \sum_{\nu \in 2_{N}} E_{\overline{\nu(n)}} .
$$

From this, it can be shown that $P$ is a perfect subset of $E$. Thus we have accomplished the proof of Theorem 2 .

Remark 1. $P$ is in the class $C\left(\sum_{1}^{1}\right)_{\rho \sigma}$. For, since $M_{n}$ is recursive in $O$ there is a number $e$ such that

$$
w \in M_{n} \equiv U\left(\mu y T_{2}^{1}(\bar{\tau}(y), e, n, w)\right)=0,
$$

where $\tau$ is the representing function of $O$. Then $P$ can be expressed in the following form:

$$
\alpha \in P \equiv(n)(k)\left[\alpha \in \delta(k) \rightarrow \delta(k) \oplus M_{n}\right],
$$

where $\delta(k) \in M_{n} \equiv(\exists y)\left[T_{2}^{1}(\bar{\tau}(y), e, n, \delta(k)) \wedge U(y)=0\right]$

$\equiv(\exists y)(\exists u)\left[u=\prod_{i<y} p_{i}^{(u) i} \wedge T_{2}^{1}(u, e, n, \delta(k)) \wedge U(y)=0\right.$

$\left.\wedge(i)_{i<y}\left\{\left(i \in O \rightarrow(u)_{i}=1\right) \wedge\left(i \notin O \rightarrow(n)_{i}=2\right)\right\}\right]$.

$\delta(k) \in M_{n}$ is a $\left(\sum_{1}^{1}\right)_{\rho \sigma}$-predicate. Hence $P$ belongs to the class $C\left(\sum_{1}^{1}\right)_{\rho \sigma}$ by (23).

3.4. Now let us prove the following

Theorem 3. There is a non-denumerable $\sum_{1}^{1}$-set in $N^{N}$ which contains no non-empty $\Pi_{1}^{1}$-subset.

Proof. Let

$$
V(\alpha, n) \equiv(\beta)(\exists x) T_{0}^{\alpha, \beta}(n, x) .
$$

For every $\Pi_{1}^{1}$-predicate $P(\alpha)$ (of a single function-variable $\alpha$ ) there is an $n$ such that

$$
(\alpha)[P(\alpha) \equiv V(\alpha, n)] .
$$

By the effective choice principle (with a number-parameter $n$ ), from each set $\hat{\alpha} V(\alpha, n)$ we can select a unit subset $\hat{\alpha} V_{1}(\alpha, n)$ such that $\operatorname{ran} V_{1}(\alpha, n)$ is also a $\Pi_{1}^{1}$-predicate. ${ }^{12)}$ Let $W$ be the set defined as

12) Of course, for $n$ such that $\overline{(\exists \alpha)} V(\alpha, n), \hat{\alpha} V_{1}(\hat{\alpha}, n)$ is empty. 
follows :

$$
\alpha \in W \equiv(\exists n) V_{1}(\alpha, n) .
$$

Then $W$ is a $\Pi_{1}^{1}$-set and intersects with every non-void $\Pi_{1}^{1}$-set in $N^{N}$. Consequently, the complement $W^{\prime}=C W$ is a $\sum_{1}^{1}$-set and it contains no non-void $\Pi_{1}^{1}$-subset. (The set $W$ is similar to a simple set of natural numbers in the sense of Post [25].) Further $W^{\prime}$ is nondenumerable, since $W$ is denumerable. (Q.E.D.)

Corollary 1. A set $P$ given in Theorem 2 cannot, in general, be in the class $\Pi_{1}^{1}$.

Problem 1. Let $E$ be a given non-denumerable $\sum_{1}^{1}$-set in $N^{N}$. Can we obtain a perfect subset $P$ of $E$ which is in the class $\sum_{1}^{1}$ ? (Cf. Remark 2 below.)

By means of the Uniformization Theorem for $\Pi_{1}^{1}$-sets (see $\S 1.2$ ), it is well-known that every $\sum_{2}^{1}$-set in the space $X \times Y$ (where $X=$ $Y=N^{N}$ ) can be uniformized by a $\sum_{1}^{1}$-set (Kondô [11], Sampei [27」). Hence by the method of the proof of Theorem 3 we can obtain a nondenumerable $\Pi_{2}^{1}$-set in $N^{N}$ such that it contains no non-empty $\sum_{2}^{1}$-subset. Further, under Gödel's axiom of constructibility, for each $n \geq 3$ every $\sum_{1}^{n}$-set in $X \times Y$ can be made uniform by a $\sum_{n}^{1}$-set, by using a result of Addison [4;p.351]. (This also is a well-known fact.) Thus we obtain the

Theorem 4. For each $n \geq 2$, there is a non-denumerable $\Pi_{n}^{1}$-sei in $N^{N}$ such that it does not contain any non-empty $\sum_{n}^{1}$-subsets, where we assume the axiom of constructibility for the case $n \geq 3$.

3.5. Let $E$ be a subset of $N^{N}$. We denote the set of all condensation points of $E$ contained in $E$ by $E_{c}$. Let $E_{r}=E-E_{c}$.

Theorem 5. If $E$ is a $\sum_{1}^{1}$ set in $N^{N}$, then $E_{c}$ is also a $\sum_{1}^{1}$-set and hence $E_{r}$ is in the class $\left(\sum_{1}^{1}\right)_{\rho}$.

In fact, $\alpha \in E_{c}$ can be expressed in the following form:

$$
\alpha \in E_{c} \equiv \alpha \in E \wedge(n)\left[\alpha \in \delta(n) \rightarrow|\delta(n) \cap E|>*_{0}\right] .
$$

By Theorem $1|\delta(n) \cap E|>\aleph_{0}$ is $\sum_{1}^{1}$, and hence so is $E_{c}$. (Q.E.D.) 
Remark 2. As long as we concern with sets in the space $N^{N}$, Theorem 5 implies Kreisel's [17; Theorem 1]. Because, further if $E$ is closed, then $E_{c}=E_{p}$ (=the perfect kernel of $E$ ). Since a set is $\Pi_{1}^{1}$-closed in the sense of Kreisel if and only if it is a $\sum_{1}^{1}$-set and is closed, $E_{p}$ is $\Pi_{1}^{1}$-closed.

Theorem 6. There is a $\sum_{1}^{1}$-set $E$ such that the $E_{r}$ is not in the class $\mathrm{C}\left(\sum_{1}^{1}\right)_{\rho}$.

Proof. Let $A$ and $B$ be $\sum_{1}^{1}$-sets of natural numbers such that $A-B$ is not in the class $C\left(\sum_{1}^{1}\right)_{\rho}{ }^{13)}$ Let

$$
\begin{aligned}
& \alpha \in A^{*} \equiv \alpha(0) \in A \wedge(x)[\alpha(x+1)=0] \text { and } \\
& \alpha \in B^{*} \equiv \alpha(0) \in B \wedge(x)[\alpha(x+1)=0] .
\end{aligned}
$$

Then $A^{*}-B^{*}$ is a denumerable set in $N^{N}$ and is not in the class $C\left(\sum_{1}^{1}\right)_{\rho}$. We define $E$ as follows:

$$
E=A^{*}+M,
$$

where $\beta \in M \equiv \beta(0) \in B \wedge \beta(1)=0$. Obviously $E$ is $\sum_{1}^{1}$ and $E_{r}=A^{*}-B^{*}$. Hence $E$ is a desired set.

Theorem 7. If $E$ is a non-empty denumerable $\sum_{1}^{1}$-set in $N^{N}$ then the members of $E$ can be enumerated by a $\Delta_{2}^{1}$-function. ${ }^{14}$

Proof. For any non-empty set $E$ it holds

$$
|E| \leq \star_{0} \equiv(\exists \alpha) Q(\alpha),
$$

where $Q(\alpha) \equiv(\beta)[\beta \in E \rightarrow(\exists n)(x)(\beta(x)=\alpha(n, x))]$. Since $E$ is a $\sum_{1}^{1-}$ set, we can select a $\Delta_{2}^{1}$-function $\gamma_{0}$ from the set $E$ by using (D) in \$2.4. On the other hand, the $\hat{\alpha} Q(\alpha)$ is a non-empty $\Pi_{1}^{1}$-set. Hence by the effective choice principle we can select a $\Delta_{2}^{1}$-function $\alpha_{0}$ such that $Q\left(\alpha_{0}\right)$ holds. We define the function $\sigma(n, x)$ as follows:

$$
\sigma(n, x)= \begin{cases}\alpha_{0}(n, x) & \text { if } \lambda x \alpha_{c}(n, x) \in E, \\ \gamma_{0}(x) & \text { otherwise. }\end{cases}
$$

Then $\sigma$ is also a $\Delta_{2}^{1}$-function and $\left\{\lambda x_{\sigma}(n, x)\right\}_{n=0,1,2, \ldots}$ enumerates the

13) Such a set A-B can be obtained by the diagonal procedure.

14) Sampei [28] also proved the same theorem. 
members of $E$. (Q.E.D.)

Theorem 7 is a refinement of a theorem of Kondô [13].

\section{§4. The measure of an arithmetical set}

4.1. Specker [30] proved that there exists a monotone increasing bounded primitive recursive sequence of rationals $\left\{a_{n}\right\}$ such that $\lim a_{n}=\mathfrak{x}$ is not a general recursive real number. We may assume Then one can find a recursive function $\varphi$ such that

$$
\left(0, a_{n}\right) \cap N^{N}=\bigcup_{k=0}^{\infty} \delta(\varphi(n, k)) .
$$

The set

$$
E=N^{N} \cap \bigcup_{n=0}^{\infty}\left(0, a_{n}\right)
$$

is recursively open and $E=N^{N} \cap(0, \mathfrak{x})$. Hence

$$
\operatorname{mes}(E)=\mathfrak{x},
$$

where "mes" denotes the Lebesgue measure. Thus we have the

Lemma 3. There is a recursively open subset of $N^{N}$ whose measure is not a general recursive real number.

But $m e s(E)$ is an arithmetical real number for every arithmetical set $E$; a fortiori for a recursively open set. Kreisel [18] showed this result for the case where $E$ is contained in the space $I$, by using the Borel-Lebesgue's theorem. Later on, the author also obtained the same result independently. Now we shall show that this result remains true when $E$ is a subset of $N^{N}$.

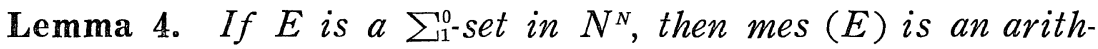
metical real number.

Proof. Let $E$ be a $\sum_{1}^{0}$-set in $N^{N}$, then there is a recursive function $\varphi$ such that

$$
E=\bigcup_{n=0}^{\infty} \delta(\varphi(n))
$$

Let $\theta$ be the function defined as follows: If $\varphi(n)=0$ for all $n$, then $\theta(m)=0$ for all $m$. If $(\exists n)[\varphi(n) \neq 0]$, 


$$
\begin{gathered}
\theta(0)=\varphi((\mu k)(n)[\delta(\varphi(k)) \neq \delta(\varphi(n)) \rightarrow \delta(\varphi(k)) \neq \delta(\varphi(n))]), \\
\theta(m+1)=\left\{\begin{array}{c}
\varphi\left(( \mu k ) \left[(i)_{i \leq m}(\delta(\varphi(k)) \cap \delta(\theta(i))=\phi) \wedge(n)\{\delta(\varphi(k))\right.\right. \\
\neq \delta(\varphi(n)) \rightarrow \delta(\varphi(k)) \nsubseteq \delta(\varphi(n))\}]) \text { if there is such a } k \\
0 \quad \text { otherwise. }
\end{array}\right.
\end{gathered}
$$

Then (i) $\theta$ is arithmetical, (ii) $\{\delta(\theta(m))\}_{m=0,1,2 \ldots}$, are disjoint each other and (iii) $E=\bigcup_{m=0}^{\infty} \delta(\theta(m))$. Hence by (ii) and (iii)

$$
\operatorname{mes}(E)=\sum_{m=0}^{\infty} m e s(\delta(\theta(m))) \text {. }
$$

Let $a_{k}=\sum_{m=0}^{k} m e s(\delta(\theta(m)))$. Then $\left\{a_{k}\right\}$ is an arithmetical sequence of rational numbers and converges. Hence the limit of $\left\{a_{k}\right\}$ is an arithmetical real number. ${ }^{15)}$ This completes the proof of Lemma 4.

By a slight modification of the proof of Lemma 4 we obtain the

Corollary 2. Let $E_{k}$ be sets in $N^{N}$ (hyper-)arithmetically open uniformly in $k$. Namely

$$
E_{k}=\bigcup_{n=0}^{\infty} \delta(\varphi(n, k))
$$

for some (hyper-) arithmetical function $\varphi$. Then $\left\{m e s\left(E_{k}\right)\right\}$ is a (hyper-) arithmetical sequence of real numbers.

4.2. Lemma 5. Every arithmetical set $E$ in $N^{N}$ can be expressed in the following form:

$$
\alpha \in E \equiv\left(*_{t} m_{t}\right)\left(*_{t-1} m_{t-1}\right) \cdots\left(m_{1}\right)\left(\exists m_{0}\right)\left[\alpha \in \delta\left(\varphi\left(m_{0}, m_{1}, \cdots, m_{t}\right)\right)\right],
$$

where $\varphi$ is recursive and $\left(*_{i} m_{i}\right)$ denotes $\left(\exists m_{i}\right)$ or $\left(m_{i}\right)$ according as $i$ is even or odd.

Proof is obvious. If we consider an arithmetical sequence of sets instead of a single set, then such a $\varphi$ can be obtained as an arithmetical function.

Lemma 6. For a given $k \geq 0$; let $\left\{E_{n_{1} n_{2} \ldots n_{k}}\right\}$ be a sequence defined as follows:

15) See Grzegorczyk [6; Theorem 2.11]. 
$\alpha \in E_{n_{1} n_{2} \ldots n_{k}} \equiv\left(*_{t} m_{t}\right)\left(*_{t-1} m_{t-1}\right) \cdots\left(m_{1}\right)\left(\exists m_{0}\right)\left[\alpha \in \delta\left(\varphi\left(n_{1}, \cdots, n_{k}, m_{0}\right.\right.\right.$, $\left.\left.\left.\cdots, m_{t}\right)\right)\right]$, where $\varphi$ is arithmetical. Then $\left\{m e s\left(E_{n_{1} \ldots n_{k}}\right)\right\}$ is an arithmetical $k$-fold sequence of real numbers.

Proof. We use the mathematical induction on $t$. Basis. $t=0$. This is no other than Corollary 2. Induction step. Let

$$
\alpha \in E_{n_{1} \ldots n_{k}} \equiv\left(*_{t+1} m_{t+1}\right)\left(*_{t} m_{t}\right) \cdots\left(m_{1}\right)\left(\exists m_{0}\right)\left[\alpha \in \delta \left(\varphi \left(n_{1}, \cdots, n_{k}, m_{0},\right.\right.\right.
$$
$\left.\left.\left.\cdots, m_{t+1}\right)\right)\right]$, where $\varphi$ is arithmetical. We define $E_{n_{1} \cdots n_{k}}^{(l)}$ as follows:

$$
\alpha \in E_{n_{1} \cdots n_{k}}^{(l)} \equiv\left(*_{t+1} m_{t+1}\right)_{m_{t+1} \leq l}\left(*_{t} m_{t}\right) \cdots\left(m_{1}\right)\left(\exists m_{0}\right)\left[\alpha \in \delta \left(\varphi \left(n_{1}, \cdots,\right.\right.\right.
$$

$\left.\left.\left.n_{k}, m_{0}, \cdots, m_{t+1}\right)\right)\right]$.

Obviously

$$
\operatorname{mes}\left(E_{n_{1} \cdots n_{k}}\right)=\lim _{l \rightarrow \infty} \operatorname{mes}\left(E_{n_{1} \cdots n_{k}}^{l}\right) .
$$

By advancing $\left(*_{t} m_{t}\right)$ in (1) and by contracting adjacent quantifiers of the same kind, we can find an arithmetical function $\theta$ such that

$$
\begin{aligned}
\alpha \in E_{n_{1} \cdots n_{k}}^{(l)} \equiv( & \left(*_{t} m_{t}\right)\left(*_{t-1} m m_{t-1}\right) \cdots\left(m_{1}\right)\left(\exists m_{0}\right) \\
& {\left[\alpha \in \delta\left(\theta\left(n_{1}, \cdots, n_{k}, l, m_{0}, \cdots, m_{t}\right)\right)\right] . }
\end{aligned}
$$

By using the hypothesis of the induction (taking $\mathrm{k}+1$ instead of $k$ ) on (2), $\left\{m e s\left(E_{n_{1} \cdots n_{k}}^{(l)}\right)\right\}$ is an arithmetical $(k+1)$-fold sequence of real numbers. Hence $\lim _{l \rightarrow \infty}\left\{\operatorname{mes}\left(E_{n_{1} \cdot n_{k}}^{(l)}\right)\right\}$ is also an arithmetical $k$-fold sequence of real numbers ${ }^{15)}$. (Q.E.D.)

Corollary 3. (Cf. Kreisel [18; p. 383].) If $E$ is an arithmetical set in $N^{N}$, then mes $(E)$ is an arithmetical real numbers.

Remark 3. (i) if $P(\alpha, k)$ and $Q(\alpha, k)$ are arithmetical predicates, then the predicates

$$
\lambda k[\operatorname{mes}(\hat{\alpha} P(\alpha, k)) \equiv \operatorname{mes}(\hat{\alpha} Q(\alpha, k))]
$$

is also arithmetical.

$$
\text { Let } E_{k}=\bigcup_{n=0}^{\infty} \delta(\varphi(n, k)) \text { and } F_{k}=\bigcup_{n=0}^{\infty} \delta(\psi(n, k)) \text {, }
$$

where $\varphi, \psi$ are (hyper)arithmetical functions. Then

$$
\lambda k\left[\operatorname{mes}\left(E_{k}\right) \leqq \operatorname{mes}\left(F_{k}\right)\right]
$$


is a (hyper) arithmetical predicate.

(i) follows from Lemmas 5 and 6, and (ii) follows from Corollary 2. Remark 3 will be used below.

\section{§5. The measure of a $\sum_{1}^{1}$ set and related problems}

5.1. Let $E$ be a $\sum_{1}^{1}$-set defined as follows:

$$
\alpha \in E \equiv(\exists \beta)(k) R(\bar{\alpha}(k), \bar{\beta}(k)),
$$

where $R$ is arithmetical and satisfies (3.12)- (3.15). (See Lemma 2.) Let $E_{\bar{\beta}(k)}=\hat{\alpha} R(\bar{\alpha}(k), \bar{\beta}(k))$. Then we obtain a monotonous and arithmetical Souslin's system $\left\{E_{n_{0} n_{1} \cdots n_{k-1}}\right\}$ such that each $E_{n_{0} n_{1} \cdots n_{k-1}}$ either is empty or consists of a single Baire's interval of order $k$. For any sequence number $w$, let

$$
\begin{aligned}
E^{w} & =\hat{\alpha}(\exists \beta)(j) R(\bar{\alpha}(\operatorname{lh}(w)+j), w * \bar{\beta}(j)) \\
& =\bigcup_{\beta \in N^{N}} \bigcap_{j=0}^{\infty} E_{w * \bar{\beta}(j) .}
\end{aligned}
$$

As is known,

$$
E=\bigcup_{n=0}^{\infty} E^{[n]} \text { and } E^{\left[n_{0}, \cdots, n_{k}\right]}=\bigcup_{n=0}^{\infty} E^{\left[n_{0}, \cdots n_{k}, n\right]} .
$$

Let $p$ be an arbitrary positive integer. We shall define a function $\beta$ inductively as follows: Step 0. By (1) there is a number $m$ such that

$$
\operatorname{mes}\left(\bigcup_{n=0}^{m} E^{[n]}\right) \geq \operatorname{mes}(E) \cdot\left(1-\frac{1}{2 p}\right) \text {. Put } \beta(0)=m \text {. Step } k+1 \text {. }
$$

Suppose $\beta(0), \cdots, \beta(k)$ are defined and

$$
\begin{aligned}
\operatorname{mes}\left(\bigcup_{n_{0}=0}^{\beta(0)} \ldots \bigcup_{n_{k}=0}^{\beta(k)} E^{\left[n_{0}, n_{1}, \cdots, n_{k}\right]}\right) \geq \operatorname{mes}(E) \cdot\left\{1-\frac{1}{p}\left(\frac{1}{2}+\frac{1}{2^{2}}+\cdots\right.\right. \\
\left.\left.+\frac{1}{2^{k+1}}\right)\right\}
\end{aligned}
$$

By (1) there is a number $m$ such that

$$
\operatorname{mes}\left(\bigcup_{n_{0}=0}^{\beta(0)} \cdots \bigcup_{n_{k}=0}^{\beta(k)} \bigcup_{n=0}^{m} E^{\left[n_{0}, \cdots, n_{k}, n\right]}\right) \geq \operatorname{mes}(E) \cdot\left\{1-\frac{1}{p}\left(\frac{1}{2}+\cdots+\frac{1}{2^{k+2}}\right)\right\} .
$$

Put $\beta(k+1)=m$. Thus 


$$
\text { (k) }\left[\operatorname{mes}\left(\bigcup_{n_{0}=0}^{\beta(0)} \cdots \bigcup_{n_{k}=0}^{\beta(k)} E^{\left[n_{0}, \cdots, n_{k}\right]}\right) \geq \operatorname{mes}(E) \cdot\left(1-\frac{1}{p}\right)\right] .
$$

Since $\bigcup_{n_{0}=0}^{\beta(0)} \ldots \bigcup_{n_{k}=0}^{\beta(k)} E^{\left[n_{0}, \cdots, n_{k}\right]} \supseteq \bigcup_{n_{0}=0}^{\beta(0)} \cdots \bigcup_{n_{k+1}=0}^{\beta(k+1)} E^{\left[n_{0}, \cdots, n_{k+1}\right]}$, we have

$$
\operatorname{mes}\left(\bigcap_{k=0}^{\infty} \bigcup_{\substack{n_{i} \leq \beta(i) \\ i \leq k}} E^{\left[n_{0}, \cdots, n_{k}\right]}\right) \geq \operatorname{mes}(E) \cdot\left(1-\frac{1}{p}\right) .
$$

Let $\Phi\langle\alpha\rangle$ be defined as follows:

$$
\Phi\langle\alpha\rangle=m e s\left(\bigcap_{k=0}^{\infty} \bigcup_{\substack{n_{i}<\alpha(i) \\ i \leq k}} E^{\left[n_{0}, \cdots, n_{k}\right]}\right) .
$$

Then obviously $(\alpha)[\Phi\langle\alpha\rangle \leq \operatorname{mes}(E)]$, and hence

$$
\sup \{\Phi\langle\alpha\rangle \mid \alpha \varepsilon N\} \leq \operatorname{mes}(E) .
$$

On the other hand, by (2)

$$
(p)_{p>0}(\exists \alpha)\left[\Phi\langle\alpha\rangle \geq \operatorname{mes}(E) \cdot\left(1-\frac{1}{p}\right)\right] .
$$

Hence it holds: $\sup \left\{\Phi\langle\alpha\rangle \mid \alpha \varepsilon N^{N}\right\} \geq \operatorname{mes}(E)$. Together with (4) we obtain

$$
\operatorname{mes}(E)=\sup \left\{\Phi\langle\alpha\rangle \mid \alpha \in N^{N}\right\} .
$$

By (3) we can see that $\Phi\langle\alpha\rangle$ is an arithmetical real number relative to $\alpha$. Indeed, it can be proved (by using the monotonity of $\left\{E_{n_{0} \cdots n_{k}}\right\}$ and König's Lemma [16]) that

$$
\bigcap_{k=0}^{\infty} \bigcup_{\substack{n_{i} \leq \alpha(2) \\ i \leq k}} E^{\left[n_{0}, \cdots n_{k}\right]}=\bigcap_{k=0}^{\infty} \bigcup_{\substack{n_{k}<\infty(i) \\ i \leq k}} E_{n_{0} n_{1} \cdots n_{k}} .
$$

(Cf. Kondô-Tugué [14: Lemma 1].) Since the set on the right-hand side of (6) is arithmetical in $\alpha$, its measure is a real number arithmetical in $\alpha$, by the relativized form of Cerollary 3. Thus we can find pair of sets $A_{\alpha}, B_{\alpha}$ consisting of rational numbers only which are arithmetical in $\alpha$ and satisfy the condition $\Phi\langle\alpha\rangle=\left(A_{\alpha}, B_{\alpha}\right)$, where $\left(A_{\alpha}, B_{\alpha}\right)$ denotes the Dedekind cut. If we put $A=\cup\left\{A_{\alpha} \mid \alpha \in N^{N}\right\}$ and $B=$ the complement of $A$ with respect to the rational numbers, then

$$
\operatorname{mes}(E)=(A, B) \text {. }
$$

Definition 1. A real number $\mathfrak{r}(\in I)$ is said to be a $\sum_{1}^{1}\left(\Pi_{1}^{1}\right.$, or 
$\Delta_{1}^{1}$ )-real number if the set of all rationals smaller than $\mathfrak{x}$ is in the class $\sum_{1}^{1}\left(\right.$ resp. $\Pi_{1}^{1}$, or $\left.\Delta_{1}^{1}\right){ }^{16)}$

Since the above set $A$ is in the class $\sum_{1}^{1}, \operatorname{mes}(E)$ is a $\sum_{1}^{1}$-real number. Thus we obtain the

Theorem 8. The measure of a $\sum_{1}^{1}$-set in $N^{N}$ is a $\sum_{1}^{1}$-real number.

Corollary 4. For every $\Pi_{1}^{1}$-set $\mathcal{E}$ in $N^{N}$, mes $(\mathcal{E})$ is a $\Pi_{1}^{1}$-real number. Consequently the measure of a $\Delta_{1}^{1}$-set is a $\Delta_{1}^{1}$-real number.

5.2. Applications of Theorem 8. 5.2.1. A refined form of a Sélivanowski theorem concerning $C A$-sets (i.e., complements of analytic sets). Let $\mathcal{E}$ be a $C A$-set in $N^{N}$, and let $\mathcal{E}_{\eta}$ 's $(\eta<\Omega)$ be the constituents of $\mathcal{E}$ with respect to a sieve determining its complement:

$$
\mathcal{E}=\bigcup_{\eta<\Omega} \mathcal{E}_{\eta}
$$

Sélivanowski [29] showed that if $m e s(\varepsilon)>0$ then there is an ordinal number $\eta_{0}<\Omega$ such that

$$
\operatorname{mes}(\mathscr{E})=\operatorname{mes}\left(\bigcup_{\eta<\eta_{0}} \mathcal{E}_{\eta}\right)
$$

For the case of a $\Pi_{1}^{1}$-set, we have the

Theorem 9. If $\mathcal{E}$ is a $\Pi_{1}^{1}$-set and mes $(\mathcal{E})>0$, then there is a $\Delta_{2}^{1}$-ordinal $\xi^{17)}$ such that

$$
\operatorname{mes}(\varepsilon)=\operatorname{mes}\left(\bigcup_{\eta<\varepsilon} \varepsilon_{\eta}\right)
$$

where $\mathcal{E}_{\eta}$ 's $(\eta<\Omega)$ are the constituents of $\mathcal{E}$ with respect to any recursive sieve determining its complement ${ }^{18)}$

Proof. Let $F$ be an arithmetical universal set for the closed sets

16) This definition for "a $\Delta_{1}^{1}$-real number" is equivalent to the other ones. (e.g., in Grzegorczyk [6], reading "hyperarithmetical" instead of "elementarily definable" there.)

17) An ordinal number $\xi$ is called a $\Delta_{2}^{1}$-ordinal, if it is the order-type of a $\Delta_{2}^{1}$-wellordering of natural numbers.

18) This theorem is an improvement of Sampei [28; Theorem 6]. And Theorem 9 of [35] is a Corollary of this Theorem 9. 
in $N^{N}$. (See $\S 6.1$ below.) We define the predicate $K(\alpha, n)$ as follows:

$$
K(\alpha, n) \equiv F^{\langle\alpha\rangle} \subseteq \mathcal{E} \wedge \operatorname{mes}\left(F^{\langle\alpha\rangle}\right) \geq \operatorname{mes}(\mathcal{E})-\frac{1}{n+1} .
$$

The first conjunction-member of the right-hand side of (8) is $\Pi_{1}^{1}$ and by Corollary 4 the second is $\sum_{1}^{1}$. Hence $K$ is $\Delta_{2}^{1}$. Since mes $(\mathcal{E})>0$, $\mathcal{E}$ contains a closed subset whose measure is arbitrarily accessible to $m e s(\mathcal{E})$. Hence it holds $(n)(\exists \alpha) K(\alpha, n)$. Consequently, we can choose a point $\alpha_{n}$ from each set $\hat{\alpha} K(\alpha, n)$ such that $\lambda n x \alpha(n, x)$ is a $\Delta_{2}^{1}$-function, where $\alpha(n, x)=\alpha_{n}(x)$. (Kondô [11]. Also cf. Sampei [28].) On the other hand, the sets $F^{\alpha_{n}>}$ are arithmetical in $\alpha$. Hence by the relativized form of Tugué-Tanaka [37; Theorem 3], there are ordinals $\xi_{n}$ recursive in $\alpha$ such that

$$
F^{\alpha_{n}>} \subseteq \bigcup_{n<\varepsilon_{n}} \mathcal{E}_{\eta} \text { for each } n \text {. }
$$

Let $\xi=\sup _{n}\left\{\xi_{n}\right\}$. Then $\xi$ is a $\Delta_{2}^{1}$-ordinal, since $\alpha$ is $\Delta_{9}^{1}$. Consequently we have (7), because for all $n$

$$
\operatorname{mes}\left(F^{\alpha_{n}}\right) \geq \operatorname{mes}(\mathcal{E})-\frac{1}{n+1} \quad \text { and } \quad F^{\alpha_{n}} \subseteq \bigcup_{n<\xi} \mathcal{E}_{n} \subseteq \mathcal{E} .
$$

This completes the proof of Theorem 9.

Problem 2. Let $\varepsilon$ be an arbitrarily given $\Pi_{1}^{1}$-subset of $N^{N}$ whose measure is positive. And let $\mathcal{E}_{\eta}$ 's be its constituents with respect to any recursive sieve determining $C \mathcal{E}$. Does it hold mes $(\mathcal{E})=$ $\operatorname{mes}\left(\bigcup_{\eta<\omega_{1}} \mathcal{E}_{\eta}\right)$ ? Here $\omega_{1}$ denotes the first non-constructive ordinal.*)

5.2.2. The following theorem is an effective version of KondôTugué's theorem [14].

Theorem 10. Let $E$ be a set in the class $\Sigma_{1}^{1}$ (or $\left.\Pi_{1}^{1}\right)$ in $N^{N} \times$ $N^{N}$. Then the set

$$
\Gamma(E)=\left\{a \mid \operatorname{mes}\left(E^{\langle\alpha\rangle}\right)>0\right\}
$$

is contained in the same class.

Proof. First we remark that in the proof of Theorem 8 the con-

*) See the end of this paper. 
dition "mes $(E)>0$ " is not used. Let

$$
\operatorname{mes}\left(E^{\langle\alpha\rangle}\right)=\left(A_{\alpha}, B_{\alpha}\right) .
$$

Then by Theorem $8, A_{\alpha}$ is $\sum_{1}^{1}$ or $\Pi_{1}^{1}$ relative to $\alpha$ according as $E$ is $\sum_{1}^{1}$ or $\Pi_{1}^{1}$. Further we have

$$
\Gamma(\exists)=\left\{\alpha \mid(\exists r)\left[r>0 \wedge r \in A_{\alpha}\right]\right\},
$$

where $r$ is a variable ranging over the rational numbers. Hence $\Gamma(E)$ is $\sum_{1}^{1}$ or $\Pi_{1}^{1}$ according as $E$ is so.

5.3. Definition 2. A real number $\mathfrak{x}$ is called a $\Delta_{1}^{1}$-real number if there is a $\Delta_{1}^{1}$-function $\alpha$ such that

$$
\mathfrak{x}=(-1)^{\alpha(0)}[(\alpha(0)+1) / 2]+\sum_{n=1}^{\infty} 2^{-\left(n+\sum_{i=1}^{n} \alpha(\imath)\right) .}
$$

We denote this expression by " $\alpha$ det $r$ ".

A real number is a $\Delta_{1}^{1}$-real number if and only if it is a $\Delta_{1}^{1}$-real number in the sense of Definition 1.

Let $S$ be a set of natural numbers which is $\sum_{1}^{1}$ but not $\Pi_{1}^{1}$. Take

$$
\mathfrak{b}=\sum_{m \equiv S} \frac{1}{2^{m+1}} \cdot{ }^{19}
$$

Then $0<\mathfrak{b}<1$ and $\mathfrak{b}$ is not a $\Delta_{1}^{1}$-real number. For, suppose $\mathfrak{b}$ were a $\Delta_{1}^{1}$-real number. Then there would be a $\Delta_{1}^{1}$-function $\beta$ suct that $\beta \operatorname{det} \mathfrak{b}$, and hence

$$
m \in S \equiv(\exists n)\left[m+1=n+\sum_{i=1}^{n} \beta(i)\right] .
$$

Consequently $S$ would be a $\Delta_{1}^{1}$-set, which is a contradiction.

Now we define the set $M(\subseteq I)$ as follows:

$$
\mathfrak{x} \in M \equiv 0<\mathfrak{x}<\mathfrak{b} .
$$

Then for any $\alpha$ and $\mathfrak{x}$ it holds:

$$
\text { If } \alpha \operatorname{det} \mathfrak{x} \text {, then }
$$

(10) $\mathfrak{x} \in M \equiv \alpha(0)=0 \wedge(\exists k)\left[k>0 \wedge(n)\left\{0<n<k \rightarrow n+\sum_{i=1}^{n} \alpha(i)-1 \in S\right\}\right.$

19) The experession (9) is not unique only if $S$ either is finite or it contains eventually, that is, from a certain number on, all natural numbers. But since $S$ is a proper $\sum_{1}^{1}$-set, it is not the case. 


$$
\wedge(\exists m)\left(m \in S \wedge k-1+\sum_{i=1}^{k \cdot 1} \alpha(i)<m+1<k+\sum_{i=1}^{k} \alpha(i)\right) .
$$

By $A(\alpha)$ we denote the right-hand member of (10). Since $S$ is $\sum_{1}^{1}$, $A(\alpha)$ is also $\sum_{1}^{1}$. Let $M^{*}=M \cap N^{N}$. Then

$$
\beta \in M^{*} \equiv A(\Psi\langle\beta\rangle)
$$

where $\Psi$ is the arithmetical functional defined as follows:

$$
\Psi\langle\beta\rangle(0)=0 \wedge \frac{1}{\mid \beta(0)+1}+\frac{1}{\mid \beta(1)+1}+\cdots=\sum_{n=1}^{\infty} 2^{-\left(n+\sum_{i=1}^{n} \Psi\langle(\beta)(i))\right.} .
$$

Hence $M^{*}$ is a $\sum_{1}^{1}$-subset of $N^{N}$. Further we have

$$
\operatorname{mes}\left(M^{*}\right)=\operatorname{mes}(M)=\mathfrak{b} \text {. }
$$

That is, mes $\left(M^{*}\right)$ is not a $\Delta_{1}^{1}$-real number. Thus we obtain the

Theorem 11. There is a $\sum_{1}^{1}$-subset of $N^{N}$ whose measure is not a $\Delta_{1}^{1}$-real number, and hence, is not a $\Pi_{1}^{1}$-real number.

5.4. A "massgleiche Huille" of a $\sum_{1}^{1}$-set. Sampei [28] proved that a $G_{\delta}$-set including a given $\sum_{1}^{1}$-set and having the same measure with it can be defined by a $\Delta_{3}^{1}$-predicate. We can improve this result, thus:

Such a $G_{\delta}$-set can be defined by a $\Delta_{2}^{1}$-predicate.

Proof. Let $G\left(\subseteq N^{N} \times N^{N}\right)$ be a universal set for the $G_{\delta}$-sets in $N^{N}$. We may assume $G$ is arithmetical. Then $m e s\left(G^{\alpha}\right)$ is an arithmetical real number relative to $\alpha .^{20)}$ Now let $M$ be the set defined as follows:

$$
\alpha \in M \equiv \operatorname{mes}\left(G^{\langle\alpha\rangle}\right)=\operatorname{mes}(E) \wedge(\beta)\left[\beta \in E \rightarrow \beta \in G^{\langle\alpha}\right] .
$$

By using Theorem 8 it is seen that $M$ is $\Delta_{2}^{1}$. Since $M \neq \phi$, we can find a $\Delta_{2}^{1}$-function $\alpha_{0}$ contained in $M$ (Kondô [11]. Also cf. Sampei [28]). Hence $G^{i \alpha_{0}}$ is a $\Delta_{2}^{1}$-set, which is a desired one. (Q.E.D.)

\section{§6. $\Pi_{1}^{1}$-sets containing perfect subsets}

6.1. A universal set for the perfect sets in $N^{N}$. Let $\beta^{\#}$ be

20) Note that even if $(\exists \alpha)\left[G^{\langle\alpha\rangle}=\phi\right]$ this fact remains true. 
a variable ranging over $2^{N}$. We shall identify $\beta^{\#}$ with the following real number:

$$
\frac{\beta^{\#}(0)}{2}+\frac{\beta^{\sharp}(1)}{2^{2}}+\cdots+\frac{\beta^{\#}(k)}{2^{k+1}}+\cdots .
$$

Let $F$ be the set in $N^{N} \times I$ defined as follows:

$$
\left\langle\alpha, \beta^{\sharp}\right\rangle \in F \equiv(n)\left[\beta^{\sharp} \notin I_{\alpha(n)}\right] .
$$

(For $I_{k}$, see §1.1.) Then $F$ is a universal set for the closed sets in $I$, namely

(i) $(\alpha)\left[F^{\prime \alpha\rangle}\right.$ is closed in $\left.I\right]$ and

(ii) For every closed set $S$ in $I$ there is an $\alpha \in N^{N}$ such that $F^{\langle\alpha\rangle}=S$, where $F^{\prime \alpha\rangle}=\left\{\beta^{\sharp} \mid\left\langle\alpha, \beta^{\sharp}\right\rangle \in F\right\}$.

Since $F^{\prime \alpha\rangle}$ is arithmetical relative to $\alpha$, so is the predicate " $F^{\prime \alpha\rangle}$ is prefect", by Kreisel $[17 ;$ Lemma A]. Further we define the set $V$ as follows:

$$
\langle\alpha, \beta\rangle \in V \equiv\left\{F^{\langle\alpha\rangle} \text { is perfect in } I\right\} \rightarrow \Psi^{\prime}\langle\beta\rangle \in F^{\langle\alpha\rangle},
$$

where $\Psi^{\prime}\langle\beta\rangle$ is the arithmetical functional such that

$$
\begin{aligned}
(n)\left[\Psi^{\prime}\langle\beta\rangle(n) \leq 1\right] \wedge \frac{1}{\mid \beta(0)+1} \mid+\frac{1}{\mid \beta(1)+1} & +\cdots=\frac{\Psi^{\prime}\langle\beta\rangle(0)}{2} \\
& +\frac{\Psi^{\prime}\langle\beta\rangle(1)}{2^{2}}+\cdots .
\end{aligned}
$$

Lemma 7. The set $V$ is an arithmetical universal set for the perfect sets in $N^{N}$.

Proof. (i) $V^{\alpha \curlywedge}$ is either $N^{N}$ or the intersection of $N^{N}$ with a perfect subset of $I$. Obviously $V^{\alpha}$ is not empty ${ }^{1}$. Since the Baire's space $N^{N}$ is regarded as a subspace of $I, V^{\alpha}$ is perfect in $N^{N}$.

(ii) Let $S$ be an arbitrary perfect subset of $N^{N}$. Then the closure $\bar{S}$ in the space $I$ is perfect in $I$. And there is an $\alpha$ such that $\bar{S}=$ $F^{\langle\alpha\rangle}$. For this $\alpha$ it holds $S=V^{\langle\alpha\rangle}$. (Q.E.D.)

6.2. Lusin [24] proved that one can effectively choose a perfect 
subset in a $C A$-set having a non-denumerable constituent. For the case of a $\Pi_{1}^{1}$-set, we obtain the

Theorem 12. Let $\mathcal{E}$ be a $\Pi_{1}^{1}$-subset of $N^{N}$. Suppose $\mathcal{E}$ contains a perfect subset. Then there is a perfect subset $P$ of $\mathcal{E}$ such that (i) it is a $\Delta_{2}^{1}$-set, ${ }^{21)}$ and

(ii) any recursive sieve determining $C \mathcal{E}$ can be bounded on the set $P$ by a $\Delta_{2}^{1}$-ordinal. ${ }^{22)}$

Proof. Let $M$ be defined as follows:

$$
\alpha \in M \equiv(\beta)[\langle\alpha, \beta\rangle \varepsilon V \rightarrow \beta \in \mathcal{E}],
$$

where $V$ is the arithmetical universal set for the perfect sets given in $\S 6.1$. Since $M$ is $\Pi_{1}^{1}$ and is not empty by the supposition, we can select a function $\alpha_{0} \in M$ such that it is a $\Delta_{2}^{1}$-function (the effective choice principle).

Let $P=V^{\left.\alpha_{0}\right\rangle}$. The $P$ is a perfect subset of $\mathcal{E}$. Since $V$ is arithmetical, $P$ is a $\Delta_{2}^{1}$-set. This proves (i). Next we shall prove (ii). By the definition of $V$, we have

$$
C P=\bigcup_{n=0}^{\infty}\left\{\beta \mid \Psi^{\prime}\langle\beta\rangle \in I_{\alpha_{0}(n)}\right\},
$$

where $\Psi^{\prime}$ is the arithmetical functional defined in $\S 6.1$. On the other hand, there is an arithmetical functional $Z\langle\alpha\rangle$ which satisfies the following conditions:

$$
\bigcup_{n=0}^{\infty}\left\{\beta \mid \Psi^{\prime}\langle\beta\rangle \in I_{\alpha(n)}\right\}=\bigcup_{n=0}^{\infty} \delta(Z\langle\alpha\rangle(n)) .
$$

Hence

$$
C P=\bigcup_{n=0}^{\infty} \delta\left(Z\left\langle\alpha_{0}\right\rangle(n)\right) .
$$

Put $\gamma=Z\left\langle\alpha_{0}\right\rangle$. Then $r$ is a $\Delta_{2}^{1}$-function. By (1) we can find a predicate $R^{\gamma}(\beta, n)$ recursive in $\gamma$ such that

$$
\beta \in P \equiv(n) R^{\gamma}(\beta, n) .
$$

21) Sampei [28; Theorem 2] obtained the same result.

22) By (ii) it is seen that there is an $\Delta_{2}^{1}$-ordinal $\eta$ such that $\varepsilon_{\eta}$ is non-denumerable. This is Corollary 2 of Sampei [28]. 
Since $P$ is disjoint with the $\sum_{1}^{1}$ set $C \mathcal{E}$, by Tugué-Tanaka [37; Theorem 3] there are numbers $e$ and $a$ such that

( $\beta$ ) $\left[e \in W^{\beta}\right]$ ( $W$ is the set defined in Spector [31]),

(4) $a \in O^{\gamma}$,

(5) ( $\beta)\left[\beta \in P \rightarrow|e|^{\beta}<|a|^{\gamma}\right]$, and

(6) $e$ is a uniform Gödel number of the well-ordering of the section $\Gamma^{\prime \beta\rangle}$ of the recursive sieve $\Gamma$ determining $C \mathcal{E}$, from $\beta$. Thus, $P \subseteq \bigcup_{\eta<|a| r} \mathcal{E}_{\eta}$. Hence $\Gamma$ can be bounded on the set $P$ by a $\Delta_{2}^{1}$-ordinal $|a|^{\gamma}$. This proves (ii).

\section{§. Concerning a basis result for the arithmetical sets}

7.1. For any subset $E \subseteq N^{N}$ and $\varphi \in N^{N}, \varphi$ is said to be a covering of $E$ (denoted by " $\varphi \in C v(E)$ ") if $E \subseteq \bigcup_{n=0}^{\infty} \delta(\varphi(n))$. Let

$$
\varphi \in A C v(E) \equiv \varphi \in A \wedge \varphi \in C v(E),
$$

where $\mathcal{A}$ is the set of all arithmetical 1-place number-theoretic functions. Such a $\varphi$ is said to be a arithmetical covering of $E$.

Definition 3. For any $E \subseteq N^{N}$,

$$
m e s^{*}(E)=\inf _{\varphi \in A C v(E)} m e s\left(\bigcup_{n=0}^{\infty} \delta(\varphi(n))\right)
$$

where mes is the Lebesgue measure.

Lemma 8. mes* has the following properties:

$$
\begin{aligned}
& 0 \leq \text { mes }^{*}(E) \leq 1 \text { for every } E \subseteq N^{N} \text {, and mes } \text { m }^{*}(\phi)=0 . \\
& A \subseteq C \rightarrow \text { mes }^{*}(A) \leq \text { mes }^{*}(B) . \\
& \text { mes }^{*}(A \cup B) \leq \text { mes }^{*}(A)+\operatorname{mes}^{*}(B) . \\
& \text { If } E \text { is measurable, then mes }(E) \leq \operatorname{mes}^{*}(E) .
\end{aligned}
$$

Proof is obvious.

\subsection{Lemma 9. $\operatorname{mes}^{*}(\mathcal{A})=1$.}

Proof. Suppose $m e s^{*}(\mathscr{A})<1$. Then there would be a function $\varphi \in \mathcal{A}$ such that 


$$
\begin{aligned}
& \mathcal{A} \subseteq \bigcup_{n} \delta(\varphi(n)), \text { and } \\
& \left.\operatorname{mes}\left(\bigcup_{n} \delta(n)\right)\right)<1 .
\end{aligned}
$$

By using (6) we shall prove that there is a function $\alpha \in \mathcal{A}$ such that $\alpha$ is not contained in $\bigcup_{n} \delta(\varphi(n))$. This contradicts (5).

Construction of such an $\alpha{ }^{23)}$ Step 0 . First we easily have

$$
(\exists m)\left\{\operatorname{mes}\left([m] \cap \bigcup_{n} \delta(\varphi(n))\right)<\operatorname{mes}([m])\right\} .
$$

(Here we regard the sequence number $[m]$ as the corresponding Baire's interval.) Then we can define

$$
m_{0}^{*}=(\mu m)\left[\operatorname{mes}\left([m] \cap \bigcup_{n} \delta(\varphi(n))\right)<\operatorname{mes}([m])\right] .
$$

Step $k+1$. Assume that $m_{0}^{*}, m_{1}^{*}, \cdots, m_{k}^{*}$ are all defined and

$$
\operatorname{mes}\left(\left[m_{0}^{*}, m_{1}^{*}, \cdots, m_{k}^{*}\right] \cap \bigcup_{n} \delta(\varphi(n))\right)<\operatorname{mes}\left(\left[m_{0}^{*}, m_{1}^{*}, \cdots, m_{k}^{*}\right]\right)
$$

holds. Then, similary as in the proof of (7) (using (8) instead of (6)), we obtain

$$
(\exists m)\left\{\operatorname{mes}\left(\left[m_{0}^{*}, \cdots, m_{k}^{*}, m\right] \cap \bigcup_{n} \delta(\varphi(n))\right)<\operatorname{mes}\left(\left[m_{0}^{*}, \cdots, m_{k}^{*}, m\right]\right)\right\} .
$$

Hence we can define

$$
\begin{aligned}
m_{k+1}^{*} & =(\mu m)\left[\operatorname{mes}\left(\left[m_{0}^{*}, \cdots, m_{k}^{*}, m\right] \cap \cup \delta(\varphi \underset{n}{(n)})\right)\right. \\
& \left.<\operatorname{mes}\left(\left[m_{0}^{*}, \cdots, m_{k}^{*}, m\right]\right)\right],
\end{aligned}
$$

Thus we have the sequence

$$
m_{0}^{*}, m_{1}^{*}, \cdots, m_{k}^{*}, \cdots
$$

such that for each $k(8)$ holds. Let $\alpha(k)=m_{k}^{*}$ for all $k$. By Remark 3 (ii) (in §4.2), $\alpha$ is arithmetical, i.e., $\alpha \in \mathcal{A}$. But $\alpha \notin \bigcup_{n} \delta(\varphi(n))$. For, suppose $\alpha \in \bigcup_{n} \delta(\varphi(n))$. If we take a sufficiently large integer $k$, the Baire's interval $\bar{\alpha}(k)$ is contained in $\bigcup_{n} \delta(\varphi(n))$. Hence $\bar{\alpha}(k) \cap$ $\bigcup_{n} \delta(\varphi(n))=\bar{\alpha}(k)$. Consequently we have

$$
\operatorname{mes}\left(\bar{\alpha}(k) \cap \bigcup_{n} \delta(\varphi(n))\right)=\operatorname{mes}(\bar{\alpha}(k)) .
$$

23) This construction is one simplified by a suggestion of Professor Y. Sampei. The original is rather complicated. 
This contradicts (8). Thus we have accomplished the proof of Lemma 9 .

Similarly we obtain the

Lemma 10. If $E$ is a $\sum_{1}^{0}$-set, then

$$
\operatorname{mes}^{*}(E \cap \mathcal{A})=\operatorname{mes}^{*}(E)=\operatorname{mes}(E) \text {. }
$$

7.3. Lemma 11. If $E$ is a $\Pi_{1}^{0}$-set in $N^{N}$, then

$$
\operatorname{mes} *(E \cap A) \geq \operatorname{mes}(E) \text {. }
$$

Proof. Suppose

$$
\operatorname{mes}^{*}(E \cap \mathscr{A})<\operatorname{mes}(E) .
$$

Since $C E$ is a $\sum_{1}^{0}$-set, there is a recursive function $\psi$ such that

$$
C E=\bigcup_{n} \delta(\psi(n)) \text {. }
$$

Then we have

$$
m e s^{*}(C E \cap \mathcal{A}) \leq \operatorname{mes}^{*}(C E) \leq \operatorname{mes}\left(\cup_{n} \delta(\psi(n))\right)=\operatorname{mes}(C E) .
$$

Hence by (9) and (10) together with (3) of Lemma 8, it would be true that

$$
\operatorname{mes}^{*}(\mathcal{A})<\operatorname{mes}(E)+\operatorname{mes}(C E)=1 \text {. }
$$

This contradicts Lemma 9.

Theorem 13. If $E$ is $\Pi_{1}^{0}$ and mes $(E)>0$, then $E$ contains an arithmetical point.

Proof. Since mes $(E)>0$, by Lemma 11 we obtain

$$
m e s^{*}(E \cap \mathcal{A})>0 \text {. }
$$

As by (1) of Lemma 8 mes $^{*}(\phi)=0$, we have $E \cap \mathcal{A} \neq \phi$. (Q.E.D.)

Remark 4. Kleene [10] proved that there is a recursive $K_{1}$ such that

$$
(\exists \alpha)(x) K_{1}(\bar{\alpha}(x)) \wedge \overline{(\exists \alpha)} \mathscr{H}(x) K_{1}(\bar{\alpha}(x)),
$$

where $\mathcal{H}$ denotes the set of all 1-place hyperarithmetical functions. Let $E=\hat{\alpha}(x) K_{1}(\bar{\alpha}(x))$. The set $E$ is obviously a perfect subset of $N^{N}$ (cf. Kreisel $[17 ;$ p. 625$]$ ). Hence this fact implies that there is a $\Pi_{1-}^{0}$ 
subset of $N^{N}$ which is perfect (a fortiori, has the power of the continuum) but contains no hyperarithmetical points. By our Theorem 13, it is seen that such a set $E$ has a zero-measure. But there is a $\sum_{1}^{1}$ set having a positive measure which contains no hyperarithmetical points. For example, $W^{\prime}$ defined in the proof of Theorem 3 and $N^{N}-\mathcal{H}$ are so.

We can easily extend Theorem 13 as follows:

Theorem 14. If $E$ is an arithmetically closed set and mes $(E)>0$, then $E$ contains an arithmetical point.

7.4. Now we shall turn out to prove that if $E$ is arithmetical and $m e s(E)>0$ then $E$ contains an arithmetical point.

Theorem 15. Let $\left\{E_{n_{1} \ldots n_{k}}\right\}$ be an arithemetical $k$-fold sequence of sets in $N^{N}$, where $k \geq 0$. Then there exists $a(k+1)$-fold sequence of sets $\left\{A_{n_{1} \cdots n_{k}}^{p}\right\}$ satisfying the following conditions:

$$
A_{n_{1} \cdots n_{k}}^{p}=C \bigcup_{m} \delta\left(\psi\left(n_{1}, \cdots, n_{k}, p, m\right)\right)
$$

for some arithmetical function $\psi$ (i.e. $A_{n_{1} \cdots n_{n}}^{p}$ 's are arithmetically closed uniformly).

$$
\begin{aligned}
& A_{n_{1} \cdots n_{k}}^{p} \subseteq E_{n_{1} \cdots n_{k}} \text { for all } p . \\
& \operatorname{mes}\left(E_{n_{1} \cdots n_{k}}-A_{n_{1} \cdots n_{k}}^{p}\right)<2^{-\left(n_{1}+\cdots+n_{k}+p\right)} .
\end{aligned}
$$

Proof. $1^{\circ}$ ) Let $E_{n_{1} \ldots n_{k}}$ be defined as follows:

$$
\alpha \in E_{n_{1} \cdots n_{k}} \equiv(\exists m) R\left(\alpha, n_{1}, \cdots, n_{k}, m\right),
$$

where $R$ is recursive. Then there is a recursive function $\varphi$ such that

$$
E_{n_{1} \cdots n_{k}}=\bigcup_{m} \delta\left(\varphi\left(n_{1}, \cdots, n_{k}, m\right)\right) \text {. }
$$

Define $E_{n_{1} \cdots n_{k}}^{n}$ by

$$
E_{,_{1} \cdots n_{k}}^{n}=\bigcup_{m=0}^{n} \delta\left(\varphi\left(n_{1}, \cdots, n_{k}, m\right)\right) .
$$

Then it holds:

$$
\left(n_{1}, \cdots, n_{k}\right)(p)(\exists n)\left\{\operatorname{mes}\left(E_{n_{1} \cdots n_{k}}-E_{n_{1} \cdots n_{k}}^{n}\right)<2^{-\left(n_{1}+\cdots+n_{k}+p\right)}\right\} .
$$

Since the scope of the quantifiers in (14) is arithmetical (by Remark 
3 in $\S 4)$, there is an arithmetical function $\theta$ such that

$$
\operatorname{mes}\left(E_{n_{1} \cdots n_{k}}-E_{n_{1} \ldots n_{k}}^{\theta\left(n_{1}, \cdots, n_{k}, p\right)}\right)<2^{-\left(n_{1}+\cdots n_{k}+p\right)} .
$$

Let $A_{n_{1} \cdots n_{k}}^{p}=E_{n_{1} \cdots n_{k}}^{\theta\left(n_{1}, \ldots, n_{k}, p\right)}$. Clearly $A_{n_{1} \cdots n_{k}}^{p} \subseteq E_{n_{1} \cdots n_{k}}$. As $E_{n_{1} \cdots n_{k}}^{n}$ 's are openclosed, so are $A_{n_{1} \cdots n_{k}}^{b}$ 's. Hence of course $A_{n_{1} \cdots n_{k}}^{b}$ are arithmetically closed uniformly, and by (15) we obtain (13).

$\left.2^{\circ}\right)$ Let $E_{n_{1} \cdots n_{k}}$ be defined as follows:

$$
\alpha \in E_{n_{1} \cdots n_{k}} \equiv(m) R\left(\alpha, n_{1}, \cdots, n_{k}, m\right),
$$

where $R$ is recursive. Then $E_{n_{1} \ldots n_{k}}$ are recursively closed uniformly. Define $A_{n_{1} \cdots n_{k}}^{p}=E_{n_{1} \cdots n_{k}}$.

$\left.3^{\circ}\right)$ Let $E_{n_{1} \cdots n_{k}}$ be defined as follows:

$$
\alpha \in E_{n_{1} \cdots n_{k}} \equiv\left(\exists m_{1}\right)\left(m_{2}\right) \cdots\left( \pm m_{j}\right) R\left(\alpha, n_{1}, \cdots, n_{k}, m_{1}, \cdots, m_{j}\right),
$$

where $R$ is recursive. Let

$$
\alpha \in E_{n_{1} \cdots n_{k}}^{n} \equiv\left(m_{2}\right) \cdots\left( \pm m_{j}\right) R\left(\alpha, n_{1}, \cdots, n_{k}, n, m_{2}, \cdots, m_{j}\right) .
$$

By the induction hypothesis, there is a $(k+2)$-fold sequence of sets $\left\{A_{n_{1} \ldots n_{k} n}^{p}\right\}$ such that

$$
A_{n_{1} \ldots n_{k} n}^{b} \text { are arithmetically closed uniformly, }
$$

and

$$
A_{n_{1} \cdots n_{n} n}^{p} \subseteq E_{n_{1} \cdots n_{k}}^{n} \text { for all } p \text {, }
$$

Since $E_{n_{1} \cdots n_{k}}=\bigcup_{n} E_{n_{1} \ldots n_{k}}^{n}$, we have

$$
\left(n_{1}, \cdots, n_{k}\right)(p)(\exists n)\left\{\operatorname{mes}\left(E_{n_{1} \cdots n_{k}}-\bigcup_{m=0}^{n} E_{n_{1} \cdots n_{k}}^{m}\right)<2^{-\left(n_{1}+\cdots n_{k}+p+1\right)}\right\} .
$$

As the scope of the quantifiers in (19) is arithmetical, there is an arithmetical function $\theta$ such that $\bar{n}=\theta\left(n_{1}, \cdots, n_{k}, p\right)$ and

$$
\operatorname{mes}\left(E_{n_{1} \cdots n_{k}}-\bigcup_{m=0}^{\bar{n}} E_{n_{1} \cdots n_{k}}^{m}\right)<2^{-\left(n_{1}+\cdots n_{k}+p+1\right)} .
$$

Define $A_{n_{1} \cdots n_{k}}^{p}$ as follows:

$$
A_{n_{1} \ldots n_{k}}^{b}=\cup_{m=0}^{\bar{n}} A_{n_{1} \ldots n_{k} m}^{b+2}
$$


where $\bar{n}=\theta\left(n_{1}, \cdots, n_{k}, p\right)$. Then it is seen by $(16)-(20)$ that $\left\{A_{n_{1} \cdots n_{k}}^{p}\right\}$ is the desired.

$\left.4^{\circ}\right)$ Let $E_{n_{1} \cdots n_{k}}$ be defined as follows:

$\alpha \in E_{n_{1} \cdots n_{k}} \equiv\left(m_{1}\right)\left(\exists m_{2}\right) \cdots\left( \pm m_{j}\right) R\left(\alpha, n_{1}, \cdots, n_{k}, m_{1}, \cdots, m_{j}\right)$,

where $R$ is recursive. Let

$\alpha \in E_{n_{1} \cdots n_{k}}^{n} \equiv\left(\exists m_{2}\right) \cdots\left( \pm m_{j}\right) R\left(\alpha, n_{1}, \cdots, n_{k}, n, m_{2}, \cdots, m_{j}\right)$.

Then, by the hypothesis of the induction, there is a $(k+2)$-fold sequence $\left\{A_{n_{1} \cdots n_{k} n}^{p}\right\}$ such that (16)-(18) hold. Put:

$$
A_{n_{1} \ldots n_{k}}^{p}=\bigcap_{n=0}^{\infty} A_{n_{1} \cdots n_{k} n}^{p+1} .
$$

Then $\left\{A_{n_{1} \ldots n_{k}}^{p}\right\}$ are arithmetically closed uniformly and we have:

$$
\begin{aligned}
\operatorname{mes}\left(E_{n_{1} \cdots l_{k}}-A_{n_{1} \cdots n_{k}}^{p}\right) & =\operatorname{mes}\left(\bigcup_{n=0}^{\infty}\left(\bigcap_{m=0}^{\infty} E_{n_{1} \cdots n_{k}}^{m}-A_{n_{1} \cdots n_{k} n}^{p+1}\right)\right) \\
& \leqq m e s\left(\bigcup_{n=0}^{\infty}\left(E_{n_{1} \cdots n_{k}}^{n}-A_{n_{1} n_{k} n}^{p+1}\right)\right) \\
& <\sum_{m=0}^{\infty} 2^{-\left(n_{1} \mid \cdots+n_{k}+m+p+1\right)} \\
& =2^{-\left(n_{1}+\cdots+n_{k}+p\right)}
\end{aligned}
$$

by (18).

Thus the proof of Theorem 15 is completed.

Theorem 16. If $E$ is an arithemetical set in $N^{N}$ and mes $(E)>0$, then $E$ contains an arithmetical point.

Proof. By Theorem 15 with $k=0$, there is $\left\{A^{p}\right\}$ such that $A^{p}$ are arithmetically closed uniformly, $A^{p} \subseteq E$ for all $p$, and $\operatorname{mes}\left(E-A^{p}\right)<2^{-p}$.

Since $m e s(E)>0$, there exists a positive integer $p$ such that $m e s(E)$ $>2^{-p}$. Hence by (23) we have mes $\left(A^{p}\right)>0$. Consequently by Theorem 14 and (21), $A^{p}$ contains an arithmetical point, and so does $E$ by (22). (Q.E.D.)

Remark 5. It can be easily seen that if $E$ is a finite subset of $\mathcal{A}$ then $\operatorname{mes}^{*}(E)=0$. Thence, by the proofs of Theorem 13 and 16 , 
every arithmetical set having positive measure contains an infinitely many aritmetical points. ${ }^{*}$

7.5. We write $m e s^{\sharp}(E)$ instead of $m e s^{*}(E)$ when 'arithmetical' is replaced by 'hyperarithmetical' in Definition 3. Then Lemmas 8-11 and Theorem 13 remain true when mes* is replaced by mes ${ }^{*}$ with $\mathscr{H}$ instead of $\mathcal{A}$. Theorem 14 also is true when 'arithmetical (ly)' is replaced by 'hyperarithmetical(ly)'. Thus by using suitably modified Theorem 15 and by modified Remark 5 we can obtain the

Theorem 17. If $E$ is arithmetical in a given hyperarithmetical function and mes $(E)>0$, then $E$ contains an infinitely many hyperarithmetical points.

Problem 3. Let $E$ be an arbitrarily given $\Delta_{1}^{1}$-subset of $N^{N}$ whose measure is positive. Then, does it hold $E \cap \mathscr{H} \neq \phi^{\text {?*) }}$

\section{§. On the Baire's theorem concerning a monotone well-ordered family of closed sets}

8.1. By Kreisel $\left[19 ;\right.$ p.246] we can see that every $\sum_{1}^{1}$ well-ordering of natural numbers represents a constructive ordinal. In the similar way we can easily extend this for the case of $\sum_{1}^{1}$ well-orderings on $N^{N}$, thus:

Lemma 12. Let $R(\alpha, \beta)$ be a $\sum_{1}^{1}$-predicate on $N^{N}$. If $\lambda \alpha \beta R(\alpha, \beta)$ is a well-ordering (that is, if it well-orders the field $D_{R}=\hat{\alpha}(\exists \beta)$ $[R(\alpha, \beta) \vee R(\beta, \alpha)])$, then its order-type $\tau\left(D_{R}\right)$ represents a constructive ordinal.

Proof. Following Kreisel's proof, by $\prec_{e}$ we denote the recursive binary relation of natural numbers whose Gödel number is $e$, and by $D_{e}$ we denote its field. Let $L(e)$ and $W(e)$ be the predicates " ${ }{ }_{e}$ is a linear ordering" and " $\prec_{e}$ is a well-ordering", respectively. Spector [31] proved that $L(e)$ is arithmetical and $W(e)$ is a complete $\Pi_{1}^{1}$-predicate in the sense of Post [25]. Following Kreisel, let $W_{1}(e)$ denote the predicate " $\prec_{e}$ is a linear ordering and $D_{e}$ is embedded into $D_{R}$ by an order-

\footnotetext{
*) See the end of this paper.
} 
preserving mapping". Then we have

$$
\begin{gathered}
W_{1}(e) \equiv L(e) \wedge(\exists \beta)\left[(x)\left(x \in D_{e} \rightarrow \beta_{x} \in D_{R}\right)\right. \\
\left.\wedge(x)(y)\left(x \prec_{e} y \rightarrow R\left(\beta_{x}, \beta_{y}\right)\right)\right],
\end{gathered}
$$

where $\beta_{x}(t)=\beta(J(x, t))$. Since $R$ is $\sum_{1}^{1}, W_{1}(e)$ is also in the class $\sum_{1}^{1}$. Assume that $\lambda \alpha \beta R(\alpha, \beta)$ is a well-ordering. Then clearly

$$
W_{1}(e) \rightarrow W(e) .
$$

If $\tau\left(D_{R}\right)$ were not smaller than $\omega_{1}$, where $\omega_{1}$ is the first non-constructive ordinal, then it would hold

$$
W(e) \rightarrow W_{1}(e) \text {. }
$$

By (1) and (2) we had $W(e) \equiv W_{1}(e)$. Hence $W(e)$ would be expressible in a $\sum_{1}^{1}$-form. This contradicts the above Spector's theorem. Consequently we must have $\tau\left(D_{R}\right)<\omega_{1}$. (Q.E.D.)

Corollary 5. Let $E$ be a $\sum_{1}^{1}$-set in $N^{N}$. If $E$ is well-ordered with respect to the usual linear ordering of real numbers ${ }^{3)}$, then its order-type $\tau(E)$ represents a constructive ordinal.

Proof. Put $R(\alpha, \beta) \equiv \alpha, \beta \in E \wedge \alpha<\beta .^{3)}$

8.2. Let $E$ be an open set in $I$. We shall define the numbertheoretic function $\zeta_{E}$ as follows: ${ }^{24}$

$$
\begin{aligned}
& \zeta_{E}(0)=(\mu n)\left[I_{n} \subseteq E\right], \\
& \zeta_{E}(k+1)= \begin{cases}(\mu n)\left[I_{[(k+1) / 2]+m+n} \subseteq E\right] \text { if } k+1 \text { is even, } \\
0 & \text { otherwise, }\end{cases}
\end{aligned}
$$

where $m=\sum_{i=0}^{k} \zeta_{E}(i)$. Let $\mathscr{F}=\left\{E_{\nu}\right\}_{\nu \equiv M}$, where $M$ is a subset of $N^{N}$. be a family of open sets in $I$.

Definition 4. $\mathscr{F}$ is called a $\sum_{1}^{1}$-family of open sets if the set $\left\{\zeta_{E_{\nu}} \mid \nu \in M\right\}$ is a $\sum_{1}^{1}$-set.

We shall give an example of $\sum_{1}^{1}$-families of open sets:

Lemma 13. Let $\Phi$ and $M$ be a hyperarithmetical functional and a $\sum_{1}^{1}$-subset of $N^{N}$, respectively. Put

24) Cf. Kuratowski [20; p. 144] 


$$
E_{\alpha}=\bigcup_{n=0}^{\infty} I_{\oplus\langle\alpha ;(n)} \quad \text { for } \alpha \in N^{N} .
$$

Then $\mathscr{F}=\left\{E_{\alpha} \mid \alpha \in M\right\}$ is a $\sum_{1}^{1}$-family of open sets.

Proof. There is a recursive function $\varphi$ such that for all $k$

$$
I_{k+1}=\bigcup_{j=0}^{\infty} \bar{I}_{\mathscr{P}(k, j)}
$$

where "-" denotes the closure operation on $I$. Then

$$
\begin{aligned}
& I_{k} \subseteq E_{\alpha} \equiv k=0 \vee\left[k>0 \wedge \bigcup_{j=0}^{\infty} \bar{I}_{g(k-1, j)} \subseteq \bigcup_{i=0}^{\infty} I_{\propto\langle\alpha\rangle(i)}\right],
\end{aligned}
$$

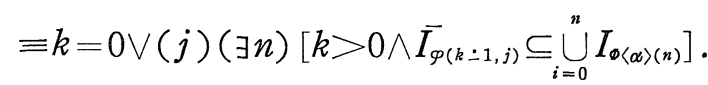

The latter equivalence is proved by using the Borel-Lebesgue's theorem. ${ }^{25)}$ Now it can be shown that " $\bar{I}_{k} \subseteq \bigcup_{i=0}^{n} I_{\alpha(i)}$ " is an arithmetical predicate of $k, n, \alpha$. Hence the $\lambda k \alpha\left[I_{k} \subseteq E_{\alpha}\right]$ is a hyperarithmetical predicate. Therefore the functional

$$
\Theta\langle\alpha\rangle=\zeta_{E_{\alpha}}
$$

is also hyperarithmetical. So, the set $\left\{\zeta_{E_{\alpha}} \mid \alpha \in M\right\}$ is a $\sum_{11}^{1}$-set in $N^{N}$, since we have

$$
\beta \in\left\{\zeta_{E_{\alpha}} \mid \alpha \in M\right\} \equiv(\exists \alpha)[\alpha \in M \wedge \beta=\Theta\langle\alpha\rangle] .
$$

8.3. Baire proved that every monotone increasing well-ordered family of open sets is denumerable. (We deal with this statement instead of what is concerned with "descending well-ordered family of closed sets".) More precisely, let $\left\{E_{\eta}\right\}(\eta<\Omega)$ be a family of open sets and let

$$
E_{\mathrm{c}} \subseteq E_{1} \subseteq \cdots \subseteq E_{\eta} \subseteq E_{\eta+1} \cdots \mid \Omega .
$$

Then there exists an ordinal $\eta_{0}<\Omega$ such that

$$
\eta \geq \eta_{0} \rightarrow E_{\eta}=E_{\eta_{0}} \text {. }
$$

Now, as an effective version of this theorem we obtain the following

Theorem 18. Let $\mathscr{F}=\left\{E_{\alpha} \mid \alpha \in M\right\}$ be a $\sum_{1}^{1}$-family of open sets

25) Cf. Lacombe [21] and Kreisel [18]. 
in I. If $\mathscr{I}$ is well-ordered by the inclusion relation $\subseteq$, then its order-type $\tau(\mathscr{F})$ represents a constructive ordinal. That is, the well-ordered sequence is stationary from a certain constructive ordinal on.

Proof. By the supposition, the set $\left\{\zeta_{E_{\alpha}} \mid \alpha \in M\right\}$ is $\sum_{1}^{1}$. And clearly we have

$$
E_{\alpha} \subseteq E_{\beta} \equiv \zeta_{E_{\alpha}} \leq \zeta_{E_{\beta}}{ }^{3,24)}
$$

Since $\mathscr{I}$ is well-ordered by $\subseteq$, the set $\left\{\zeta_{E_{\alpha}} \mid \alpha \in M\right\}$ is also well-ordered by $\leq$, and

$$
\tau\left(\left\{\zeta_{E_{\alpha}} \mid \alpha \in M\right\} ; \leq\right)=\tau(\mathscr{I} ; \subseteq) .
$$

By Corollary $5, \tau\left(\left\{\zeta_{E_{\alpha}} \mid \alpha \in M\right\} ; \leq\right)<\omega_{1}$. Consequently we have $\tau(\mathscr{I} ; \subseteq)$ $<\omega_{1}$. This completes the proof of Theorem 18.

8.4. Remark 6. As is seen above, when we concern with the space $I, \lambda k\left[I_{k} \subseteq \bigcup_{n} I_{\alpha(n)}\right]$ is arithmetical in $\alpha$. On the other hand, as far as we concern with the Baire's space $N^{N}$, the predicate $\lambda k\left[\delta(k) \subseteq \bigcup_{n}\right.$ $\delta(\alpha(n))]$ is not hyperaritmetical in $\alpha$. Because we have the

Lemma 14. There is a recursive function $\varphi$ such that $\lambda k[\delta(k)$ $\left.\subseteq \bigcup_{n} \delta(\varphi(n))\right]$ is not hyperarithmetical.

Proof. Take a recursive $K_{1}$ such that

$$
(\exists \alpha)(x) K_{1}(\bar{\alpha}(x)) \wedge(\exists \alpha) \mathscr{H}(x) K_{1}(\bar{\alpha}(x)) .
$$

Since $K_{1}$ is recursive, there is a recursive function $\varphi$ such that $G=$ $\cup \delta(\varphi(n))$, where $G=\hat{\alpha}(\exists x) \bar{K}_{1}(\bar{\alpha}(x))$. As $G \neq N^{N}$, it holds ( $\exists n$ ) $[\delta(n) \ddagger G]$. Now we shall define $\psi$ as follows:

$$
\begin{aligned}
& \psi(0)=(\mu n)[\delta(n) \subsetneq G], \\
& \psi(k+1)=(\mu n)[\delta(n) \varsubsetneqq \delta(\psi(k)) \wedge \delta(h) \varsubsetneqq G] .
\end{aligned}
$$

Clearly $\psi$ is well defined. Since

$$
\delta(n) \subsetneq \delta(m) \rightarrow \ln (\delta(n))>\operatorname{lh}(\delta(m)),
$$

$\bigcap_{n=0}^{\infty} \delta(\psi(n))$ consists of a single point which does not belong to $G$. We denote it by $\alpha_{0}$ : 


$$
\left\{\alpha_{0}\right\}=\bigcap_{n} \delta(\psi(n))
$$

Suppose " $\delta(k) \subseteq G$ " were hyperarithmetical. Then by (3) $\psi$ is also hyperarithmetical. But since

$$
\alpha_{0}(x)=y \equiv(\alpha)\{(n)[\alpha \in \delta(\psi(n))] \rightarrow \alpha(x)=y\},
$$

$\alpha_{0}$ is also hyperarithmetical. Consequently $C G=\hat{\alpha}(x) K_{1}(\bar{\alpha}(x))$ would contain a hyperarithmetical point $\alpha_{0}^{\prime}$. This contradicts the definition of $K_{1}$. Hence " $\delta(k) \subseteq G$ " cannot be hyperarithmetical. This proves Lemma 14.

On the contrary, $\lambda k\left[\delta(k) \subseteq C \bigcup_{n} \delta(\alpha(n))\right]$ is arithmetical in $\alpha$, because we have

$$
\delta(k) \subseteq C \bigcup_{n} \delta(\alpha(n)) \equiv(n)[\delta(k) \cap \delta(\alpha(n))=\phi]
$$

\section{REFERENCES}

[1] Addison, J.W., Analogies in the Borel, Lusin and Kleene hierarchies, I, II, Bull. Amer. Math. Soc. 61 (1955), 75, 171-172.

[2] Hierarchies and the axiom of constructibility, Summaries of talks presented at the Summer Institute of Symbolic Logic in 1957 at Cornell University 3 (1957), 355-362.

[3] Separation principles in the hierarchies of classical and effective descriptive set theory, Fund. Math. 46 (1958), 123-135.

[4] Some consequences of the axiom of constructibility, Fund. Math. 46 (1959), 337-357.

[5] Gandy, R. O., On a problem of Kleene's, Bull. Amer. Math. Soc. 66 (1960), 501502.

[6] Grzegorczyk, A., Elemenatrily definable analysis, Fund. Math. 41 (1954), 311338.

[7] Kleene, S. C., Introduction to metamathematics, North-Holland, Amsterdam, 1952.

[8] Arithmetical predicates and function quantifiers, Trans. Amer. Math. Soc. 79 (1955), 312-340.

[9] On, the forms of the predicates in the theory of constructive ordinals (second paper), Amer. J. Math. 77 (1955), 405-428.

[10] Hierarchies of number-theoretic predicates, Bull. Amer. Math. Soc. 61 (1955), 193-213.

[11] Kondô, M., Sur 1'uniformisation des complémentaire analytiques et les ensembles projectifs de la seconde classe, Japan. J. Math. 15 (1938), 197-230.

[12] — The theory of analytic sets (Japanese), Tokyo, 1938.

[13] On denumerable analytic sets (Japanese), Magazine of the Tokyo Buturi-Gakko, No. 567 (1939), 1-6.

[14] - et T. Tugué, Quelques cribles par rapport aux mesures, J. of Math. 1 (1952), 55-61. 
[15] Sur les ensembles nommables et le fondement de analyse mathématique, I, Japan. J. Math. 28 (1958), 1-116.

[16] König, D., Über eine Schlussweise aus dem Endlichen ins Unendliche, Acta Sci. Math. (Szeged) 3 (1927), 121-130.

[17] Kreisel, G., Analysis of the Cantor-Bendixson theorem by means of the analytic hierachy, Bull. Acad. Polon. Sci., Sér. Sci. Math. Astr. Phys. 7 (1959), 621626.

[18] _ La prédicativité, Bull. Soc. Math. France 88 (1960), 371-391.

[19] , Review of Schütte's book "Beweistheorie", J. Symbolic Logic 25 (1960), 243-249.

[20] Kuratowski, C., Topologie, I, 4th ed., PAN, Warszawa, 1958.

[21] Lacombe, D., Les ensembles récursivement ouverts ou fermés et leur applications à l'analyse récursive, C. R. Acad. Sci. Paris 245 (1957), 1040-1043.

[22] Ljapunow, A.A., E.A. Stschegolkow and W.J. Arsenin, Arbeiten zur deskriptiven Mengenlehre, Deutscher Verlag der Wissenschaften, Berlin, 1955.

[23] Lusin, N., Leçon sur les ensemble analytiques et lcurs applications, Paris, 1930.

[24] - Sur un choix d'ensemble parfail distingué dans un complémentaire analytique arbitraire ayant des constituantes non dénombrables, C. R. Acad. Sci. Paris 201 (1935), 806-809.

[25] Post, E.L., Recursive enumerable sets of positive integers and their decision problems, Bull. Amer. Math. Soc. 50 (1944), 284-316.

[26] Sampei, Y., On the uniformization of the complement of an analytic set, Comment. Math. Univ. St. Paul. 10 (1962), 58-62.

[27] - On the complete basis for the $\Delta_{2}^{1}$-sets, Ibid. 13 (1965), 81-86.

[28] - On the principle of effective choice and its applications, Ibid. 15 (1966), 29-42.

[29] Sélivanowski, E., Sur les propriétés des constituantes des ensembles analytiques, Fund. Math. 21 (1933), 20-28.

「30] Specker, E., Nicht konstruktiv beweisbare Sätze der Analysis, J. Symbolic Logic 14 (1949), 145-158.

[31] Spector, C., Recursive well-ordering, J. Symbolic Logic 20 (1955), 151-163.

[32] Suzuki, Y., On the uniformization principle, Proceedings of the Symposium on the foundations of mathematics held at Katata, Kyoto (1962), 137-144.

[33] Tanaka, H., A note on the limit of a sequence of arithmetical functionals, to appear in Proc. Amer. Math. Soc.

[34] - On limits of sequences of hyperarithmetical functionals and predicates, Comment. Math. Univ. St. Paul. 14 (1966), 105-121.

[35] , Some properties of $\sum_{1}^{1}$-and $\Pi_{1}^{1}$-sets in $N^{N}$, Proc. Japan Acad. 42 (1966), 304-307.

[36] Tugué, T., Predicates recursive in a type-2 object and Kleene hierarchies, Comment. Math. Univ. St. Paul. 8 (1959), 97-117.

[37] and H. Tanaka, A note on the effective descriptive set theory, Ibid. 15 (1966), 19-28.

[38] Watanabe, H., Une remarque sur 1'uniformisation des ensembles analytiques plans, Tôhoku Math. J. 5 (1954), 79-82. 
Note added in proof (April 21, 1967):

1) Theorem 16 was also obtained by Sacks: Measure-theoretic uniformity, Bull. Amer. Math. Soc., 73 (1967), 169-174.

2) Ppoblem 2 can be solved affirmatively as a consequence of a result of Sacks' paper mentioned in 1).

3) Recently the author obtained an affirmative answer for Problem 3. The author will write details for 2) and 3) elsewhere before long. 